\title{
Enhancing the Charge Transportation Ability of Yolk-Shell Structure for High-Rate Sodium and Potassium Storage
}

Yi Zhao, ${ }^{\dagger}$ Xiuling Shi, ${ }^{\dagger}$ Samuel Jun Hoong Ong, ${ }^{\ddagger}, \|$ Qianqian Yao,${ }^{\dagger}$ Bingbing Chen,${ }^{\perp}$

Kun Hou, ${ }^{\dagger}$ Chuntai Liu, ${ }^{\#}$ Zhichuan J. Xu, $, \$, \S, \Delta$ Lunhui Guan ${ }^{*}, \dagger$

$\nmid$ CAS Key Laboratory of Design and Assembly of Functional Nanostructures, Fujian

Key Laboratory of Nanomaterials, Fujian Institute of Research on the Structure of

Matter, Chinese Academy of Sciences, Fuzhou 350108, China

$₫$ School of Materials Science and Engineering, Nanyang Technological University, Singapore 639798, Singapore

"Singapore-HUJ Alliance for Research and Enterprise (SHARE), Nanomaterials for

Energy and Energy-Water Nexus (NEW), Campus for Research Excellence and

Technological Enterprise (CREATE), Singapore 138602

§Energy Research Institute@NTU, Nanyang Technological University, 50 Nanyang

Drive, Singapore, Singapore 639798, Singapore

${ }^{\Delta}$ Solar Fuels Lab, Nanyang Technological University, 50 Nanyang Drive, Singapore, Singapore 639798, Singapore

${ }^{\#}$ Key Laboratory of Materials Processing \& Mold (Zhengzhou University), Ministry of Education, Zhengzhou University, Zhengzhou, 450002, China

${ }^{\perp}$ Department of Energy Science and Engineering, Nanjing Tech University, Nanjing 210000, Jiangsu Province, China

*Corresponding author: guanlh@fjirsm.ac.cn 

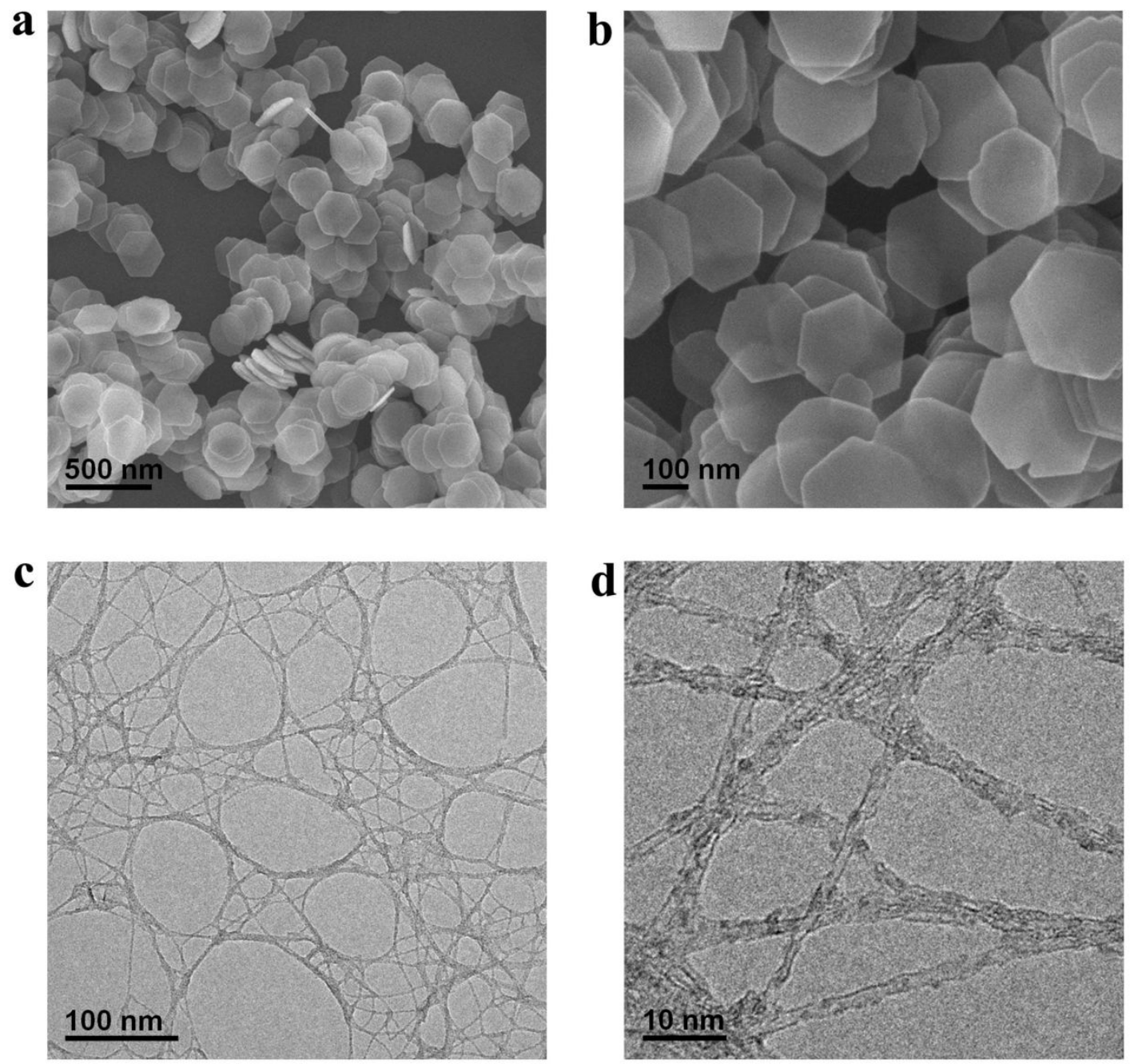

Figure S1. (a-b) SEM images of bare $\mathrm{Fe}_{2} \mathrm{O}_{3}$ nanosheets. (c-d) TEM images of individual SWNT with average diameter of $\sim 1.4 \mathrm{~nm}$ and length from several hundred $\mathrm{nm}$ to several $\mu \mathrm{m}$. 

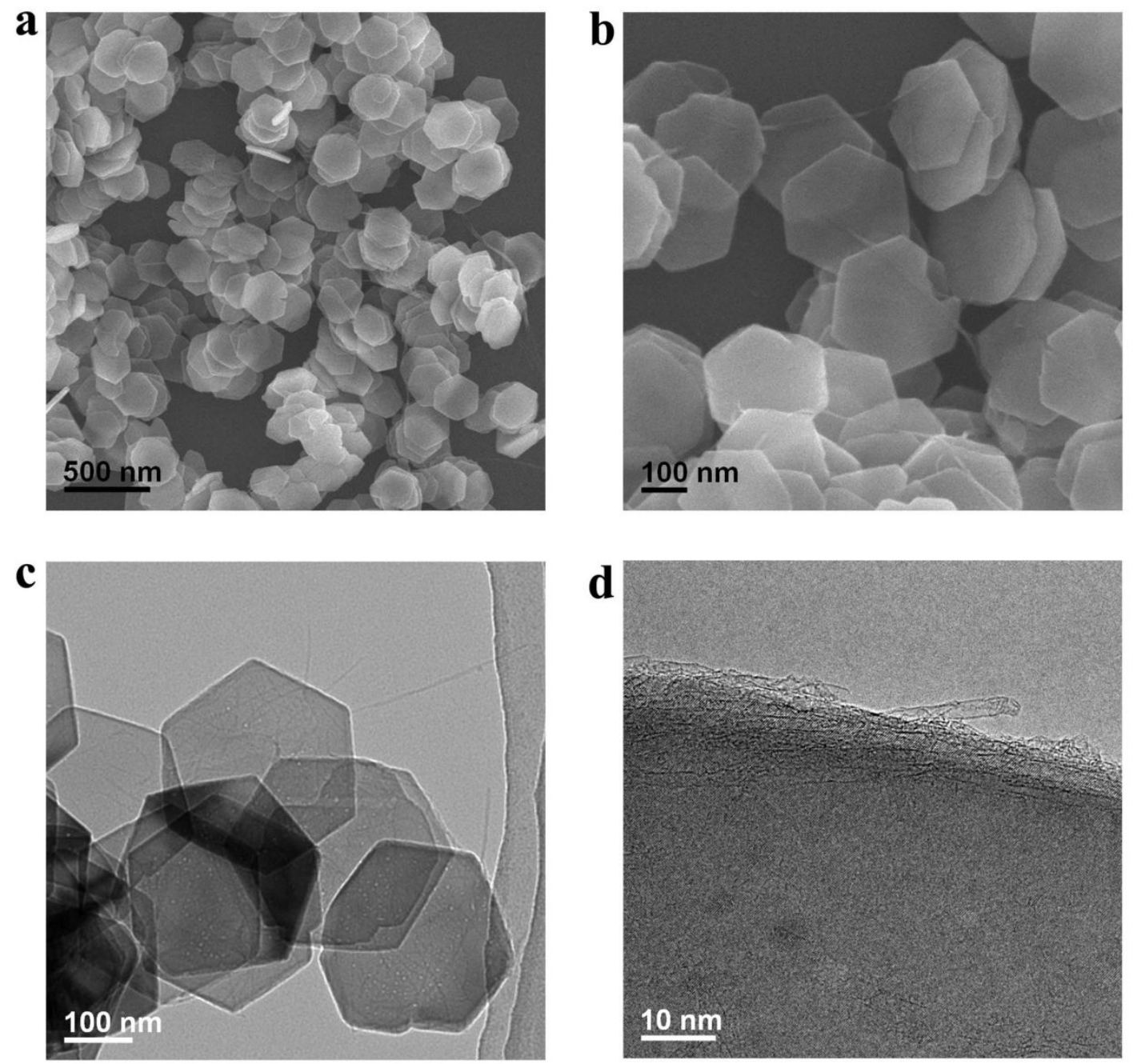

Figure S2. (a-b) SEM and (c-d) TEM images of $\mathrm{Fe}_{2} \mathrm{O}_{3} / \mathrm{SWNT}$ composite, illustrating the covering of SWNT layer on the surface of $\mathrm{Fe}_{2} \mathrm{O}_{3}$ nanosheet. 

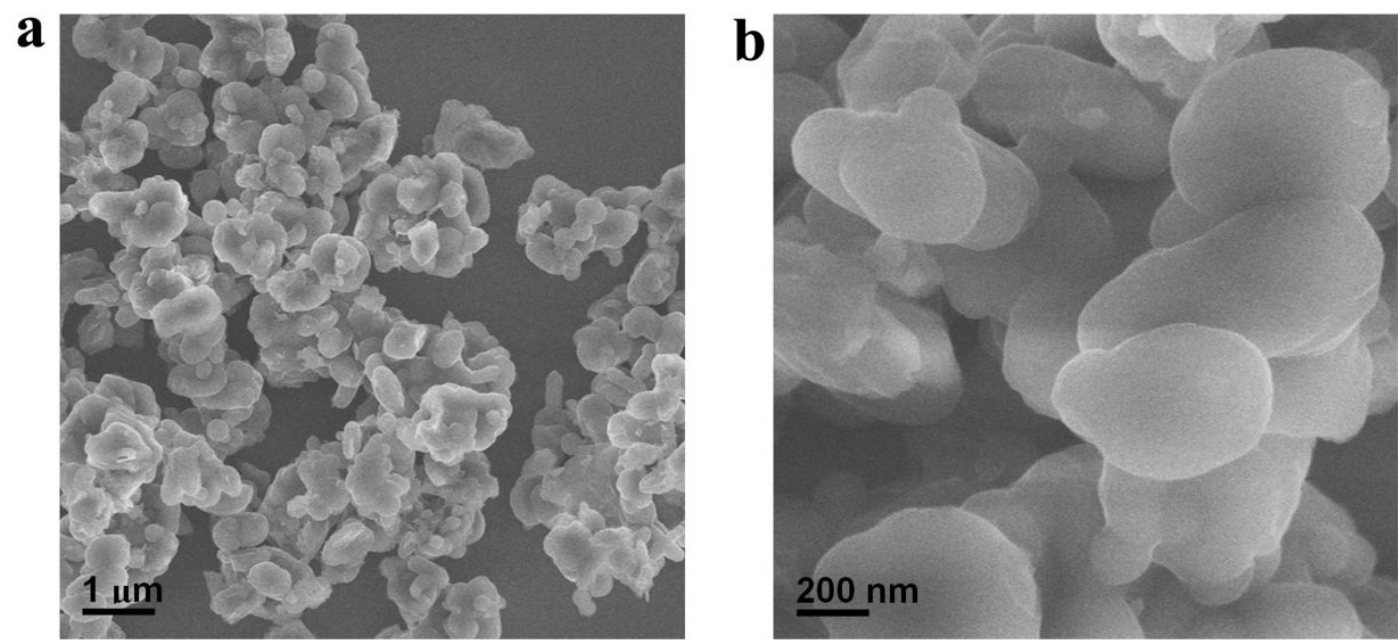

c
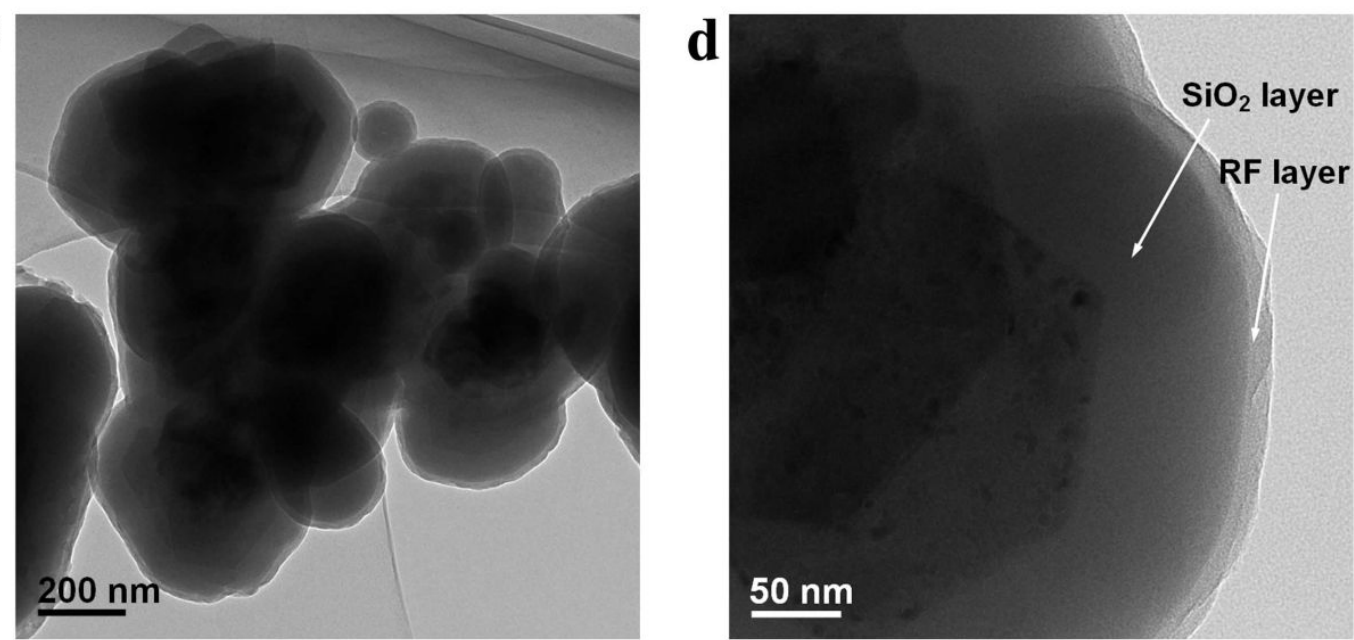

Figure S3. (a-b) SEM and (c-d) TEM images of $\mathrm{Fe}_{2} \mathrm{O}_{3} / \mathrm{SWNT} @ \mathrm{SiO}_{2} @ \mathrm{RF}$ composite, from which $\mathrm{Fe}_{2} \mathrm{O}_{3} / \mathrm{SWNT}$ composite was sequentially coated with $\mathrm{SiO}_{2}$ and RF layers. 
$\mathbf{a}$

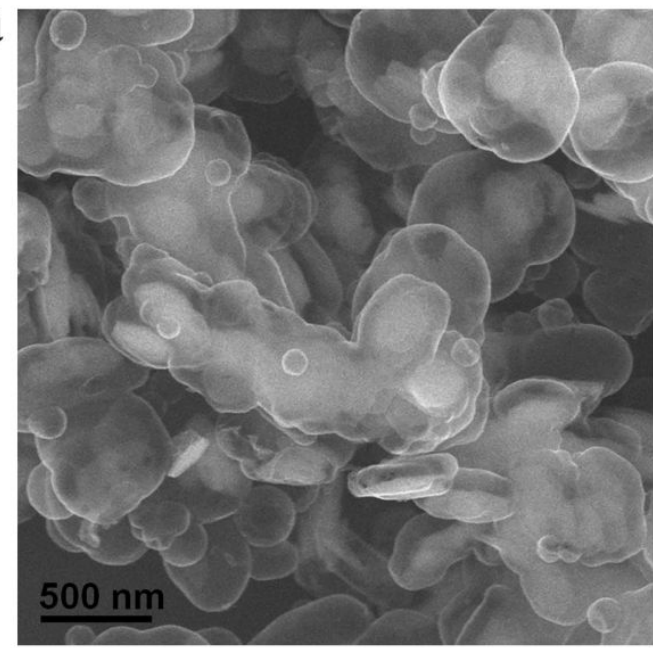

C

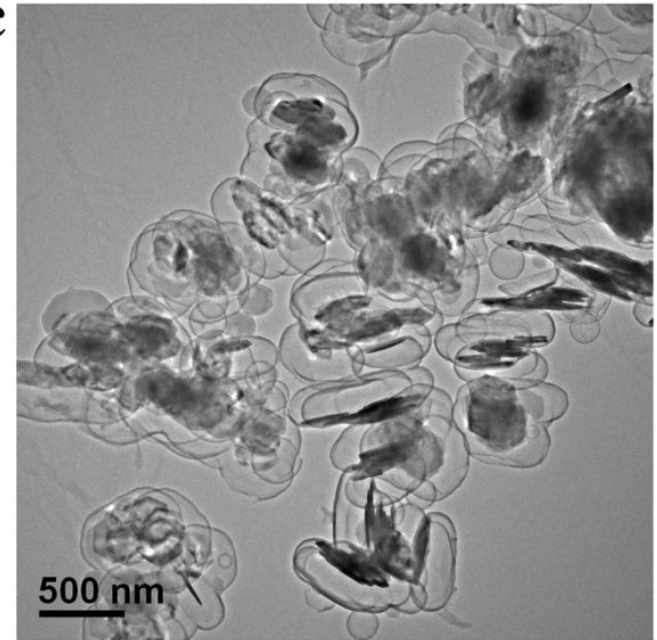

b

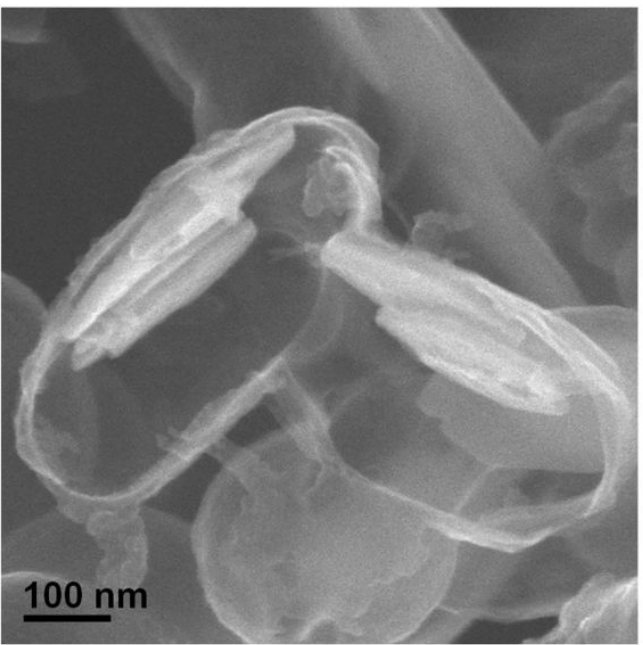

d

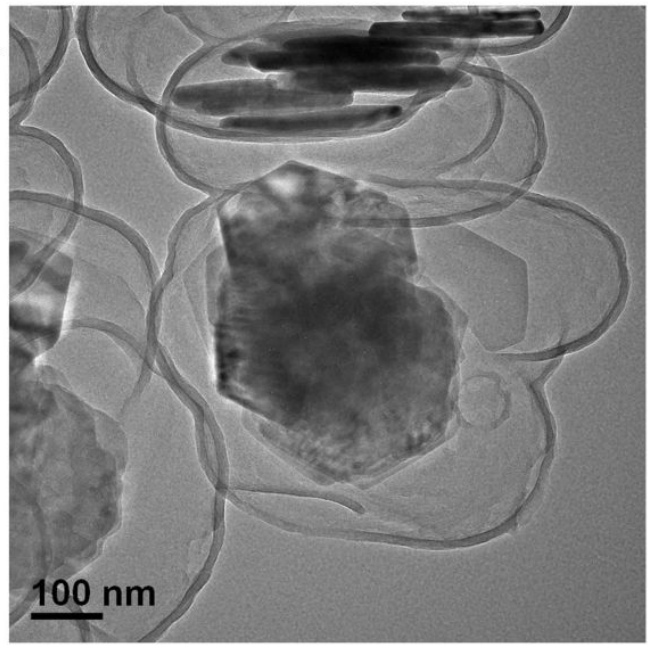

Figure S4. (a-b) SEM and (c-d) TEM images of $\mathrm{FeO}_{\mathrm{x}} / \mathrm{SWNT} @ \mathrm{C}$ composite, disclosing the formation of yolk-shell structure with large interior void space. The yolk was composed of several $\mathrm{FeO}_{\mathrm{x}}$ nanosheets, and the thickness of carbon shell was around $12-16 \mathrm{~nm}$. 


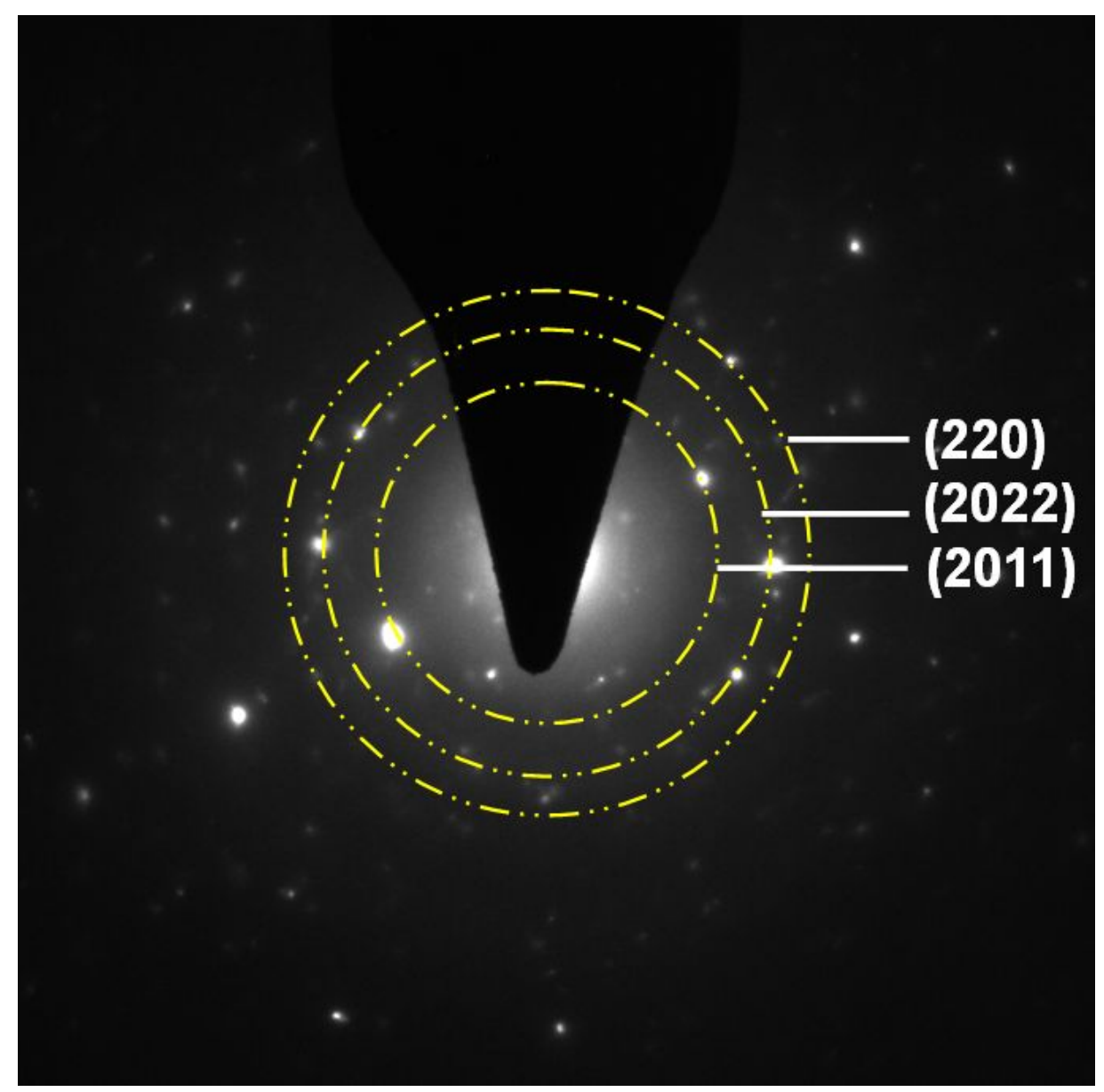

Figure S5. SAED pattern of $\mathrm{Fe}_{1-\mathrm{x}} \mathrm{S} / \mathrm{SWNT} @ \mathrm{C}$ composite. The diffraction rings can be well assigned to the (2011), (2022), and (220) planes of $\mathrm{Fe}_{1-\mathrm{x}} \mathrm{S}$. 

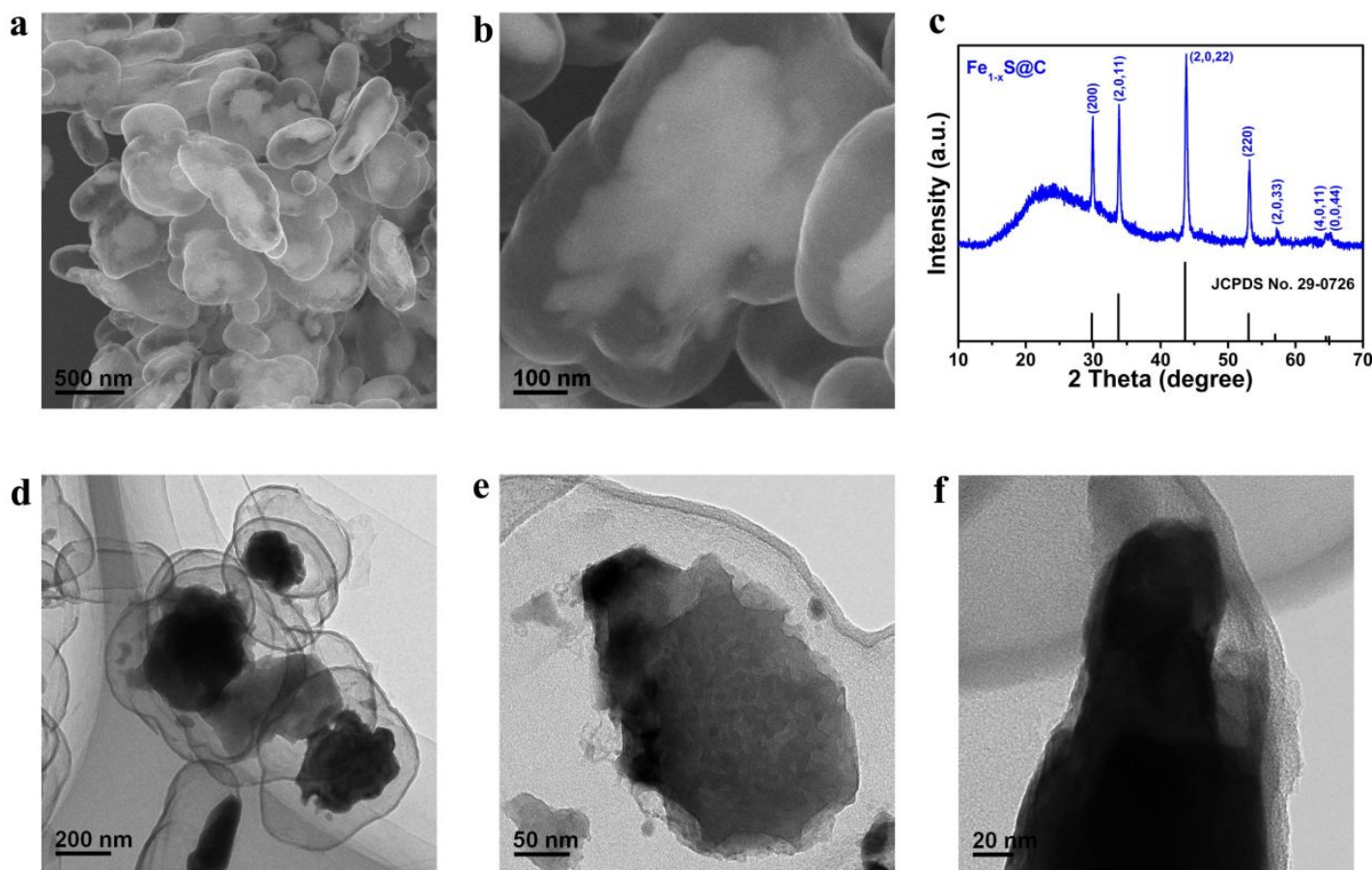

Figure S6. (a-b) SEM, (c) XRD pattern, and (d-f) TEM images of Y-S Fe $\mathrm{Fe}_{1-\mathrm{x}} \mathrm{S} @ \mathrm{C}$ composite. As can be seen, $\mathrm{Fe}_{1-\mathrm{x}} \mathrm{S}$ particle was well confined within hollow carbon nanosheet with large interior void room. SWNT channels were not detected in this composite. In Figure S6c, all the diffraction peaks can be well ascribed to hexagonal $\mathrm{Fe}_{1-\mathrm{x}} \mathrm{S}$ phase (JCPDS NO. 29-0726). 

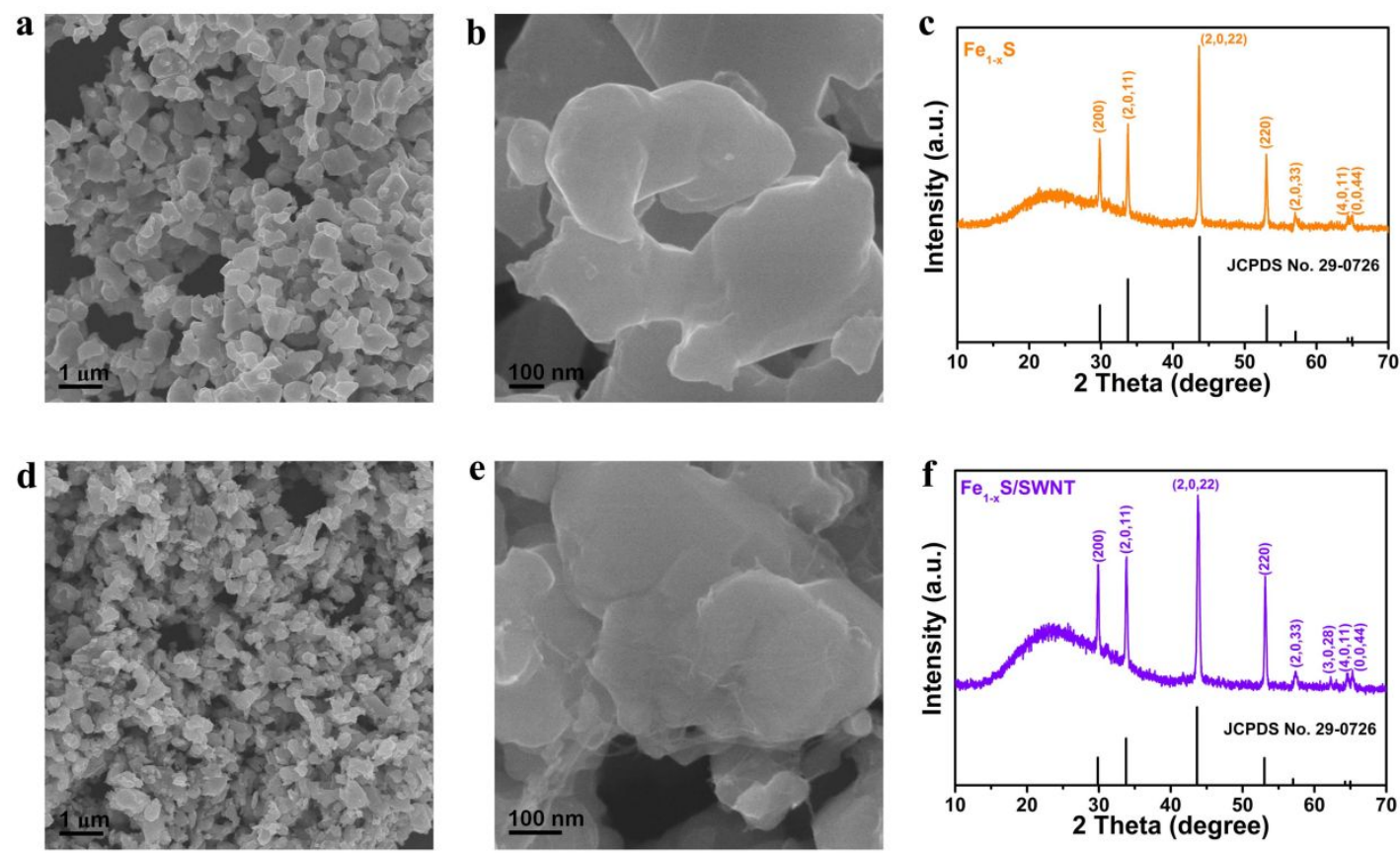

Figure S7. (a-b) SEM images and (c) XRD pattern of bare $\mathrm{Fe}_{1-\mathrm{x}} \mathrm{S}$. (d-e) SEM images and (f) XRD pattern of $\mathrm{Fe}_{1-\mathrm{x}} \mathrm{S} / \mathrm{SWNT}$ composite. The XRD pattern of these two samples displayed typical diffraction peaks of $\mathrm{Fe}_{1-\mathrm{x}} \mathrm{S}$ phase (JCPDS NO. 29-0726). Seen from the SEM images, $\mathrm{Fe}_{1-\mathrm{x}} \mathrm{S} / \mathrm{SWNT}$ and $\mathrm{Fe}_{1-\mathrm{x}} \mathrm{S}$ samples exhibited large and irregular $\mathrm{Fe}_{1-\mathrm{x}} \mathrm{S}$ particles after sulfuration treatment. For $\mathrm{Fe}_{1-\mathrm{x}} \mathrm{S} / \mathrm{SWNT}$ composite, the surface of $\mathrm{Fe}_{1-\mathrm{x}} \mathrm{S}$ particle was still covered with SWNTs. 


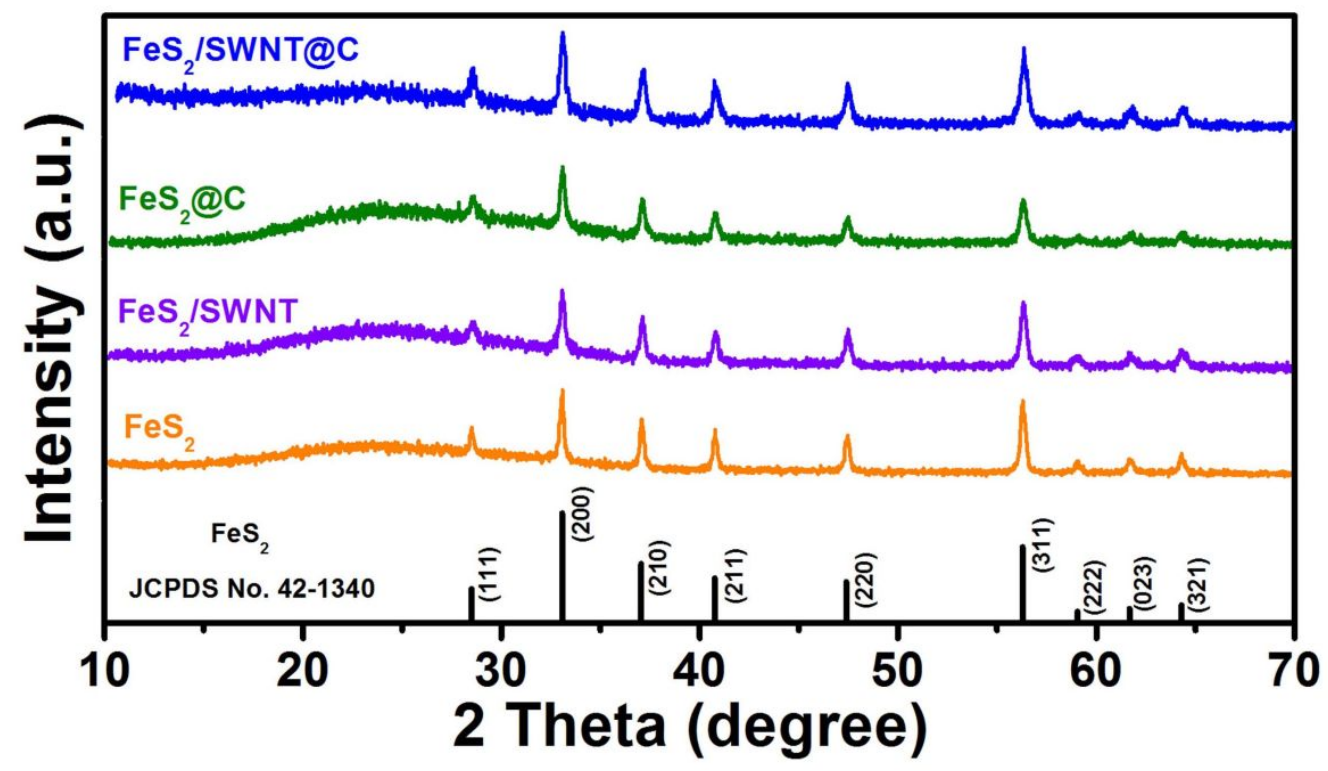

Figure S8. XRD patterns of $\mathrm{FeS}_{2}, \mathrm{FeS}_{2} / \mathrm{SWNT}, \mathrm{FeS}_{2} @ \mathrm{C}$, and $\mathrm{FeS}_{2} / \mathrm{SWNT} @ \mathrm{C}$ composites after calcination treatment at $500{ }^{\circ} \mathrm{C}$ for $3 \mathrm{~h}$ and $400{ }^{\circ} \mathrm{C}$ for $1 \mathrm{~h}$ by using $\mathrm{Fe}_{2} \mathrm{O}_{3}, \mathrm{Fe}_{2} \mathrm{O}_{3} / \mathrm{SWNT}, \mathrm{FeO}_{\mathrm{x}} @ \mathrm{C}$, and $\mathrm{FeO}_{\mathrm{x}} / \mathrm{SWNT} @ \mathrm{C}$ as precursors, respectively. As can be seen, all the XRD patterns exhibited the same diffraction peaks of pyrite $\mathrm{FeS}_{2}$ (JCPDS No. 42-1340). It demonstrated that various $\mathrm{Fe}_{2} \mathrm{O}_{3}$ and $\mathrm{FeO}_{x}$ precursors were firstly converted into same $\mathrm{FeS}_{2}$ phase during sulfuration treatment. 


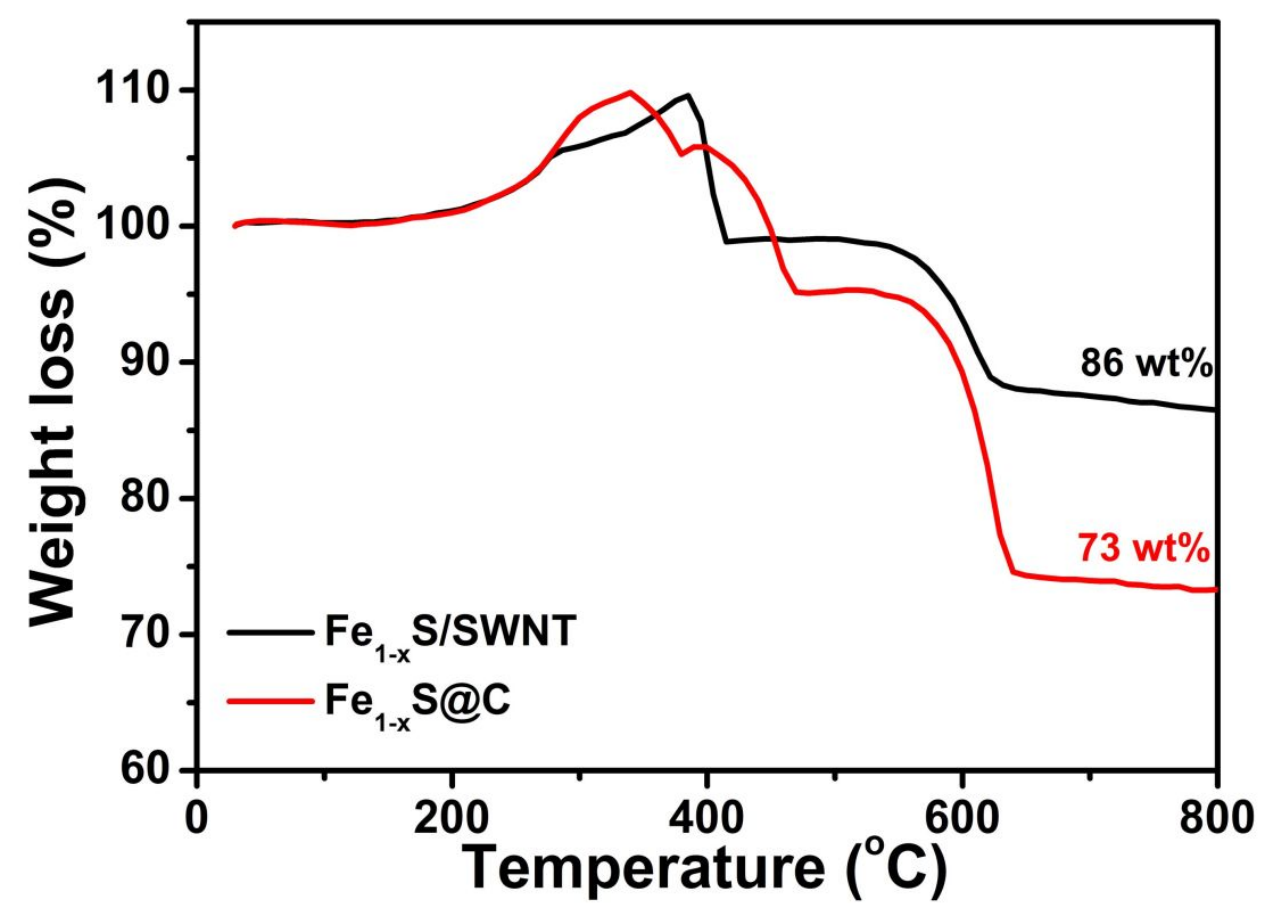

Figure S9. TGA curves of $\mathrm{Fe}_{1-\mathrm{x}} \mathrm{S} / \mathrm{SWNT}$ and $\mathrm{Fe}_{1-\mathrm{x}} \mathrm{S} @ \mathrm{C}$ composites between 30 and $800{ }^{\circ} \mathrm{C}$ in Air, which exhibited weight loss of 14 and $27 \mathrm{wt} \%$, respectively. Thus, the $\mathrm{Fe}_{1-\mathrm{x}} \mathrm{S}$ contents in $\mathrm{Fe}_{1-\mathrm{x}} \mathrm{S} / \mathrm{SWNT}$ and $\mathrm{Fe}_{1-\mathrm{x}} \mathrm{S} @ \mathrm{C}$ samples were calculated to be 98 and $83 \mathrm{wt} \%$, respectively.

Calculation details: Seen from the TGA curve of bare $\mathrm{Fe}_{1-\mathrm{x}} \mathrm{S}$, it was completely converted into $\mathrm{Fe}_{2} \mathrm{O}_{3}$ after air calcination with a weight loss of $12.5 \mathrm{wt} \%$. Thus, 100 wt $\%$ pure $\mathrm{Fe}_{1-\mathrm{x}} \mathrm{S}$ can be converted into $87.5 \mathrm{wt} \% \mathrm{Fe}_{2} \mathrm{O}_{3}$ after calcination. Based on the following Equation (1), we can deduce that the $\mathrm{x}$ value in $\mathrm{Fe}_{1-\mathrm{x}} \mathrm{S}$ phase was about 0.1 . For $\mathrm{Fe}_{1-\mathrm{x}} \mathrm{S} / \mathrm{SWNT}, \mathrm{Fe}_{1-\mathrm{x}} \mathrm{S} @ \mathrm{C}$, and $\mathrm{Fe}_{1-\mathrm{x}} \mathrm{S} / \mathrm{SWNT} @ \mathrm{C}$ composites, the final $\mathrm{Fe}_{2} \mathrm{O}_{3}$ contents after air calcination were 86, 73, and $72 \mathrm{wt} \%$, respectively. Thus, the $\mathrm{Fe}_{1-\mathrm{x}} \mathrm{S}$ contents were calculated to be 98,83 , and $82 \mathrm{wt} \%$, respectively (Equation (2)). ${ }^{\mathrm{S} 1}$

$\frac{M\left(F e_{1-x} S\right)}{m\left(F e_{1-x} S\right)}=\frac{(1-x) / 2 \times M\left(\mathrm{Fe}_{2} \mathrm{O}_{3}\right)}{m\left(\mathrm{Fe}_{2} \mathrm{O}_{3}\right)} \rightarrow \frac{56 \times(1-x)+32}{100}=\frac{(1-x) / 2 \times(56 \times 2+16 \times 3)}{87.5}$

$\frac{100}{87.5}=\frac{X}{86}=\frac{Y}{73}=\frac{Z}{72}$

(X, Y, Z are $\mathrm{Fe}_{1-\mathrm{x}} \mathrm{S}$ contents in $\mathrm{Fe}_{1-\mathrm{x}} \mathrm{S} / \mathrm{SWNT}, \mathrm{Fe}_{1-\mathrm{x}} \mathrm{S} @ \mathrm{C}$, and $\mathrm{Fe}_{1-\mathrm{x}} \mathrm{S} / \mathrm{SWNT} @ \mathrm{C}$, respectively) 

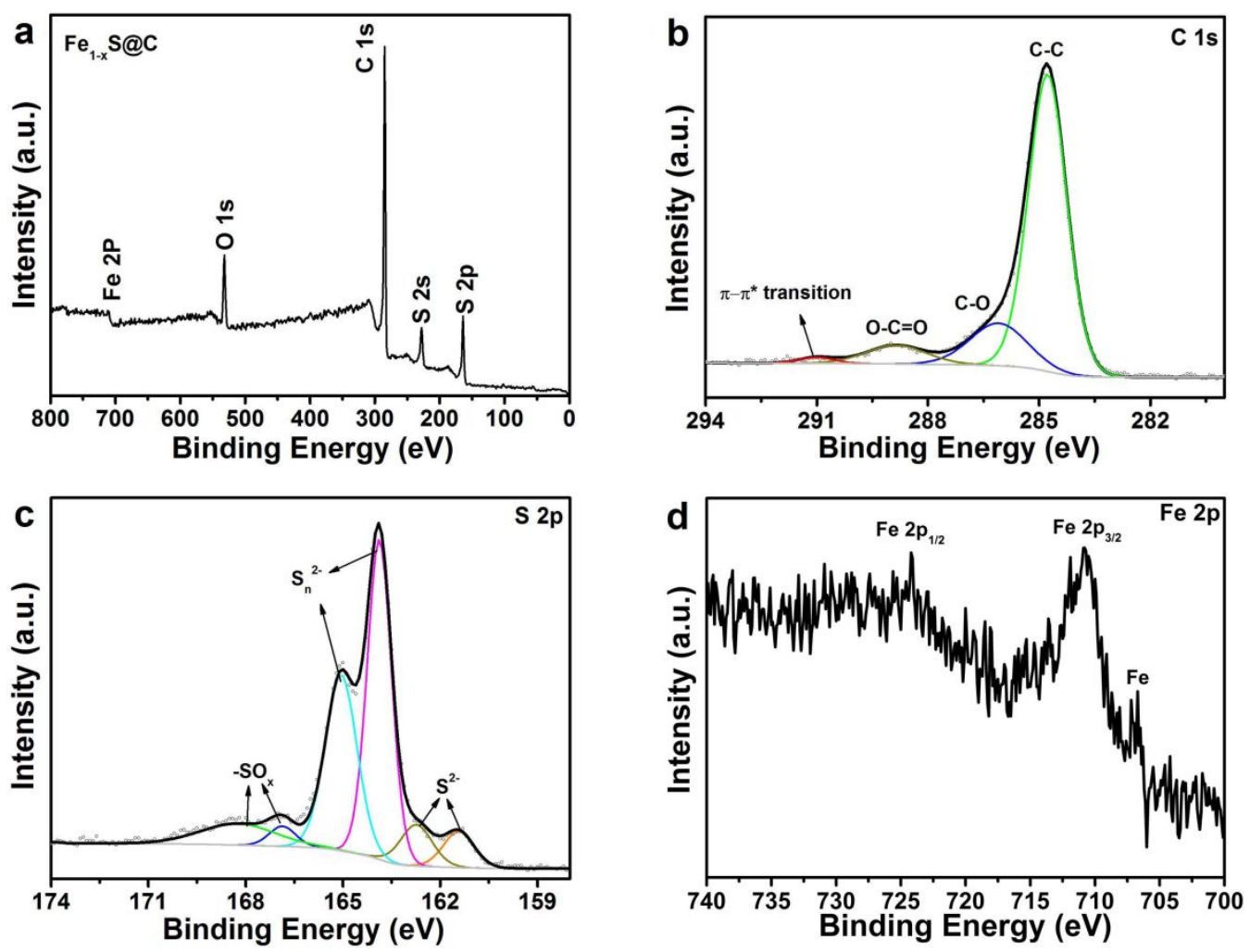

Figure S10. (a) Survey XPS spectrum of $\mathrm{Fe}_{1-\mathrm{x}} \mathrm{S} @ \mathrm{C}$ composite and its high-resolution (b) C 1s, (c) S 2p, and (d) Fe 2p XPS spectra. 

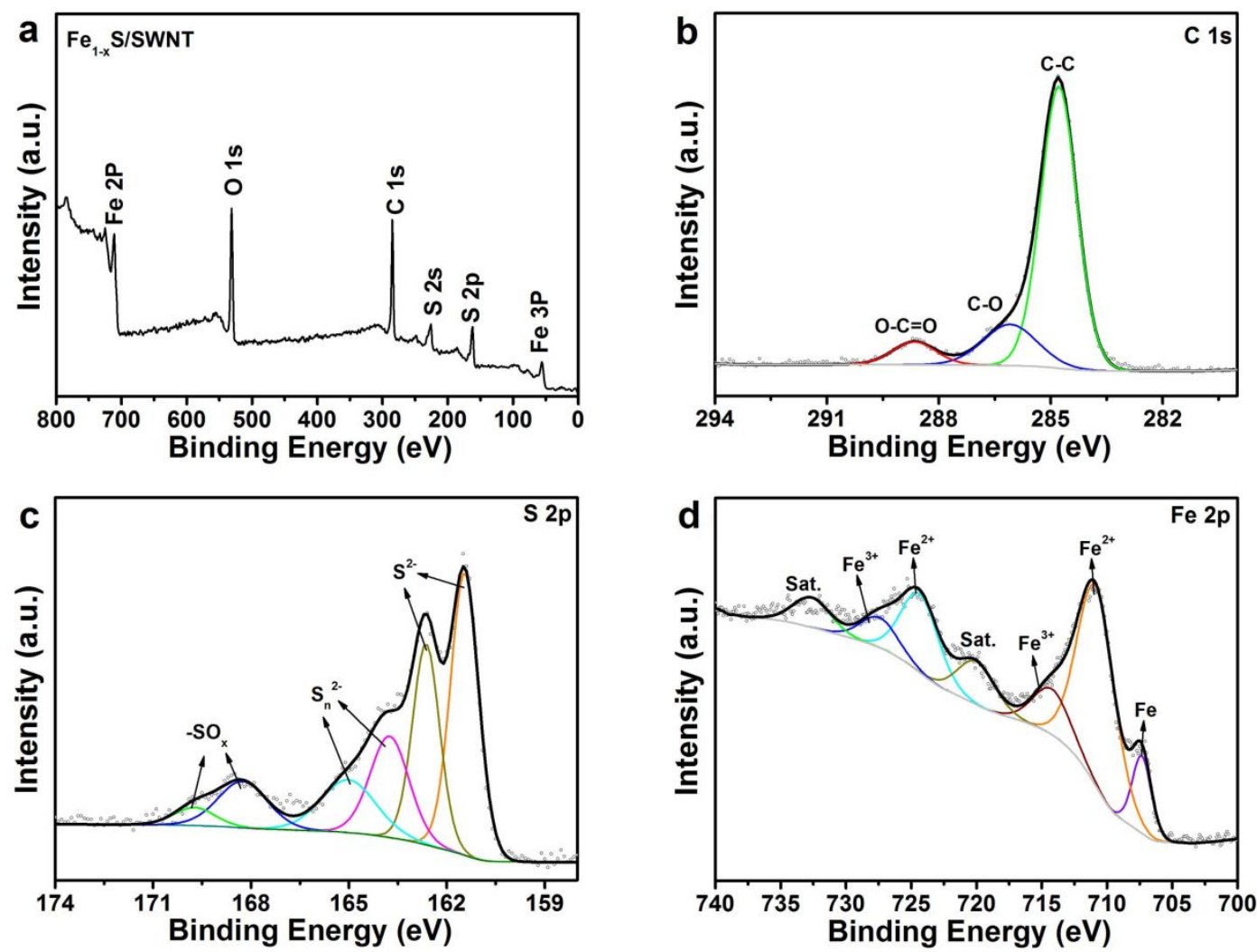

Figure S11. (a) Survey XPS spectrum of $\mathrm{Fe}_{1-\mathrm{x}} \mathrm{S} / \mathrm{SWNT}$ composite and its high-resolution (b) C 1s, (c) S 2p, and (d) Fe 2p XPS spectra. 

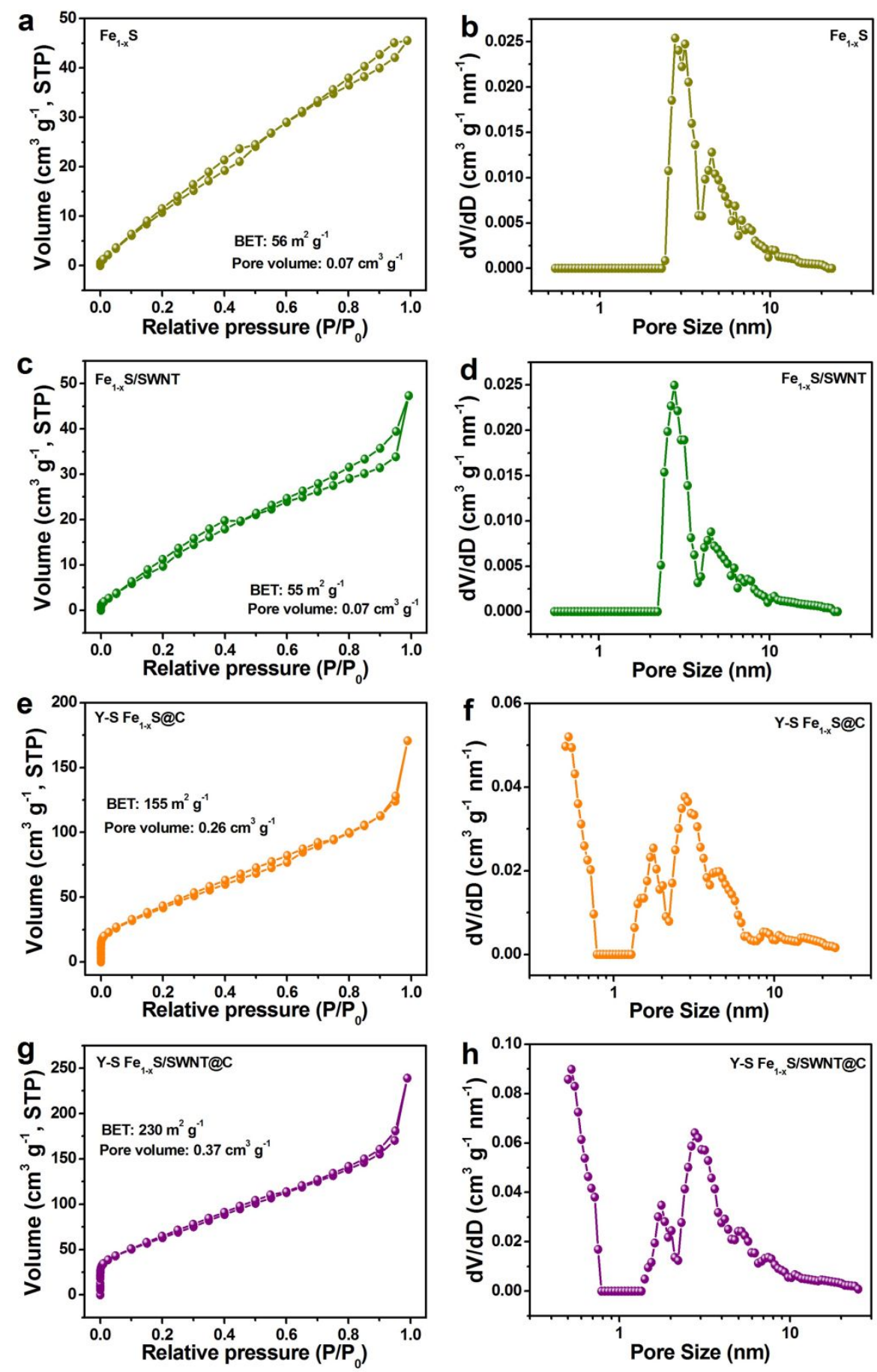

Figure S12. (a, c, e, g) Nitrogen adsorption-desorption isotherms and (b, d, f, h) pore size distribution of (a-b) $\mathrm{Fe}_{1-\mathrm{x}} \mathrm{S}$, (c-d) $\mathrm{Fe}_{1-\mathrm{x}} \mathrm{S} / \mathrm{SWNT}$, (e-f) $\mathrm{Fe}_{1-\mathrm{x}} \mathrm{S} @ \mathrm{C}$, and (g-h) $\mathrm{Fe}_{1-\mathrm{x}} \mathrm{S} / \mathrm{SWNT} @ \mathrm{C}$ composite. 


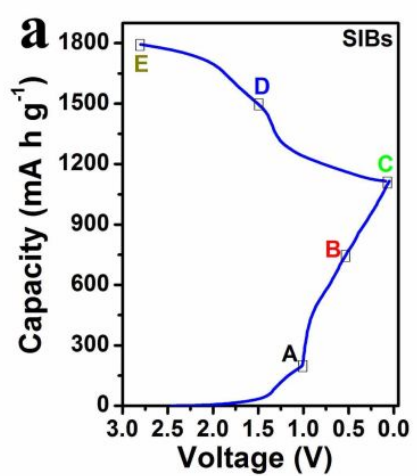

c

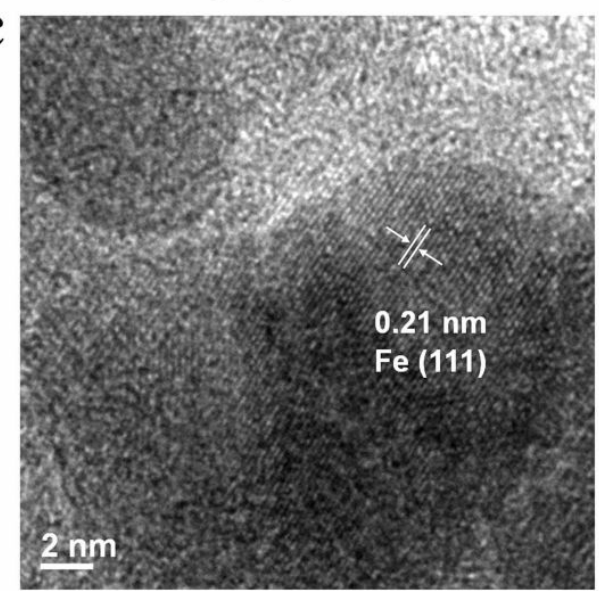

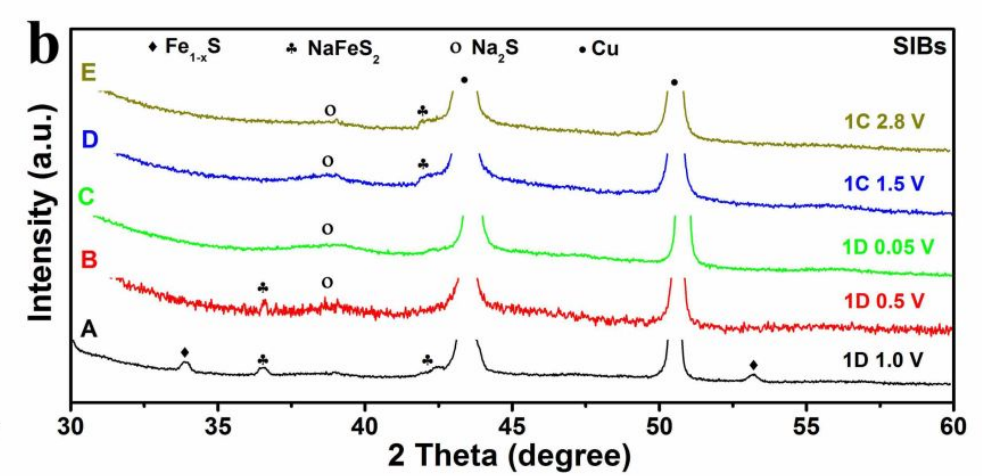

d

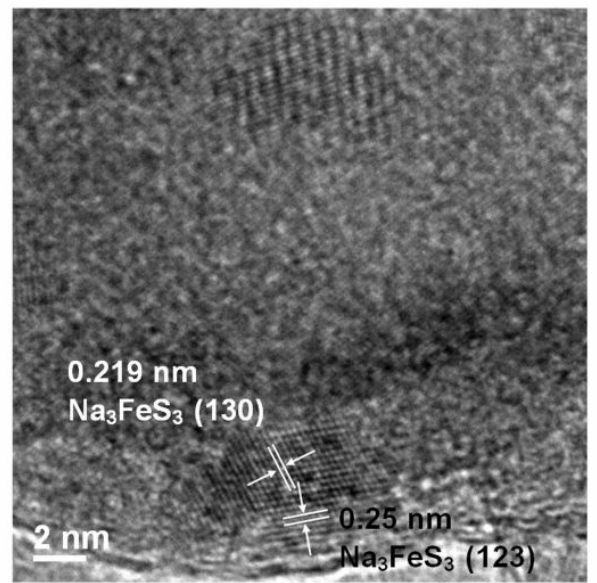

Figure S13. (a) Initial discharge/charge curves at $50 \mathrm{~mA} \mathrm{~g}^{-1}$ for SIBs, and (b) ex-situ XRD patterns of $\mathrm{Fe}_{1-\mathrm{x}} \mathrm{S} / \mathrm{SWNT} @ \mathrm{C}$ electrode under various states. HRTEM images of $\mathrm{Fe}_{1-\mathrm{x}} \mathrm{S} / \mathrm{SWNT} @ \mathrm{C}$ electrode after (c) initial discharge to $0.05 \mathrm{~V}$ and (d) after initial charge to $2.8 \mathrm{~V}$ for SIBs. In Figure $\mathrm{S} 12 \mathrm{~b}, \mathrm{NaFeS}_{2}$ phase was observed after initial discharge to $1.0 \mathrm{~V}$, illustrating the formation of Na-rich phase $\left(\mathrm{Na}_{\mathrm{y}} \mathrm{Fe}_{1-\mathrm{x}} \mathrm{S}\right)$. The peak intensity of this phase was gradually decreased and finally disappeared after discharging to $0.05 \mathrm{~V}$. When initially charged to $1.5 \mathrm{~V}$ and $2.8 \mathrm{~V}$, the peaks of $\mathrm{NaFeS}_{2}$ phase were reappeared. The ex-situ HRTEM image exhibited the lattice fringes of metallic Fe and $\mathrm{Na}_{3} \mathrm{FeS}_{3}$ after discharging to $0.05 \mathrm{~V}$ and charging to $2.8 \mathrm{~V}$, respectively. 

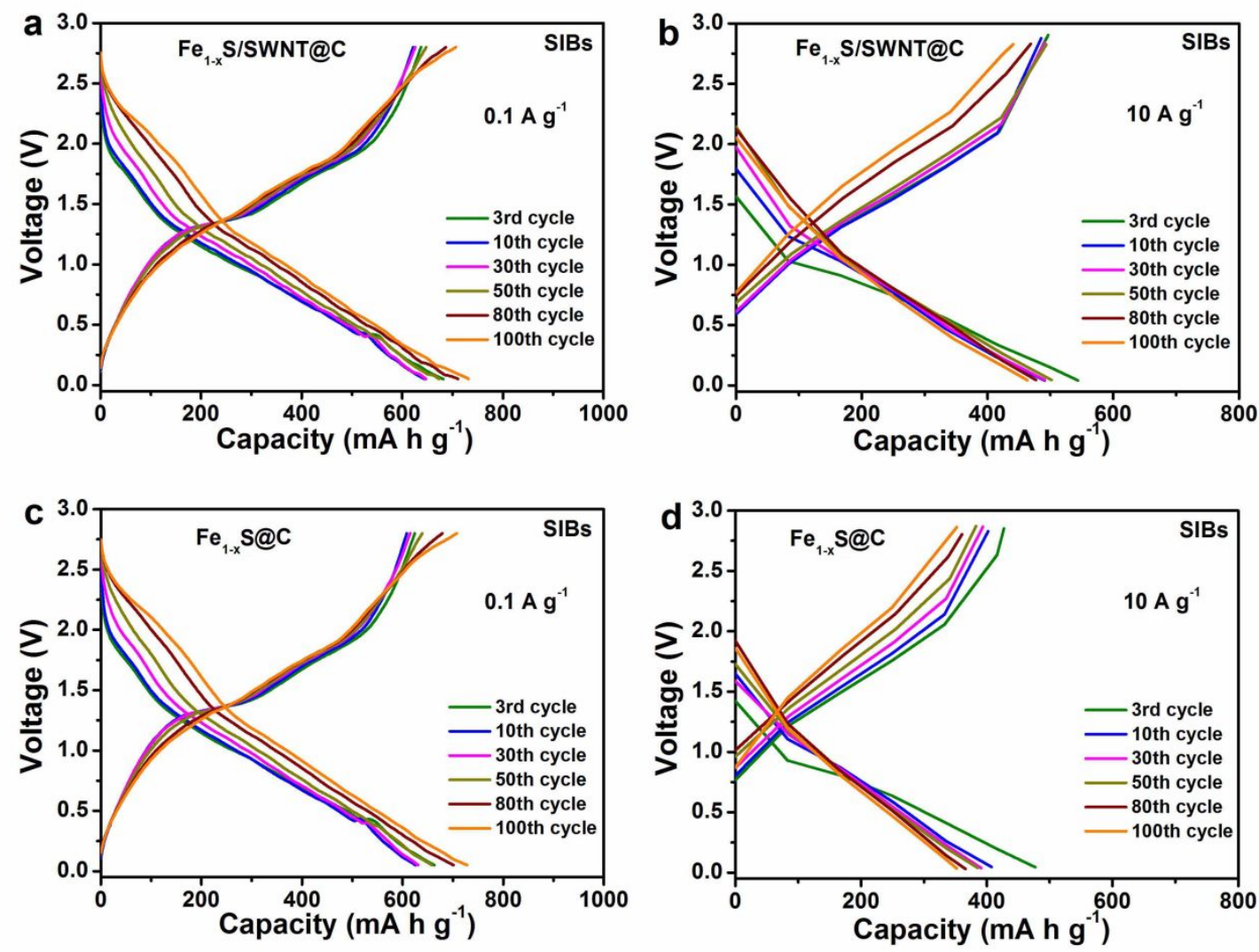

Figure S14. Selected discharge and charge profiles of (a-b) $\mathrm{Fe}_{1-\mathrm{x}} \mathrm{S} / \mathrm{SWNT} @ \mathrm{C}$ and (c-d) $\mathrm{Fe}_{1-\mathrm{x}} \mathrm{S} @ \mathrm{C}$ anodes for SIBs at (a, c) $0.1 \mathrm{~A} \mathrm{~g}^{-1}$ and $(\mathrm{b}, \mathrm{d}) 10 \mathrm{~A} \mathrm{~g}^{-1}$. As can be seen, these two composites delivered similar cycling performance at low current density of $0.1 \mathrm{~A} \mathrm{~g}^{-1}$. However, at $10 \mathrm{~A} \mathrm{~g}^{-1}, \mathrm{Fe}_{1-\mathrm{x}} \mathrm{S} / \mathrm{SWNT} @ \mathrm{C}$ anode delivered higher sodium storage capacity than $\mathrm{Fe}_{1-\mathrm{x}} \mathrm{S} @ \mathrm{C}$ under various cycles. 

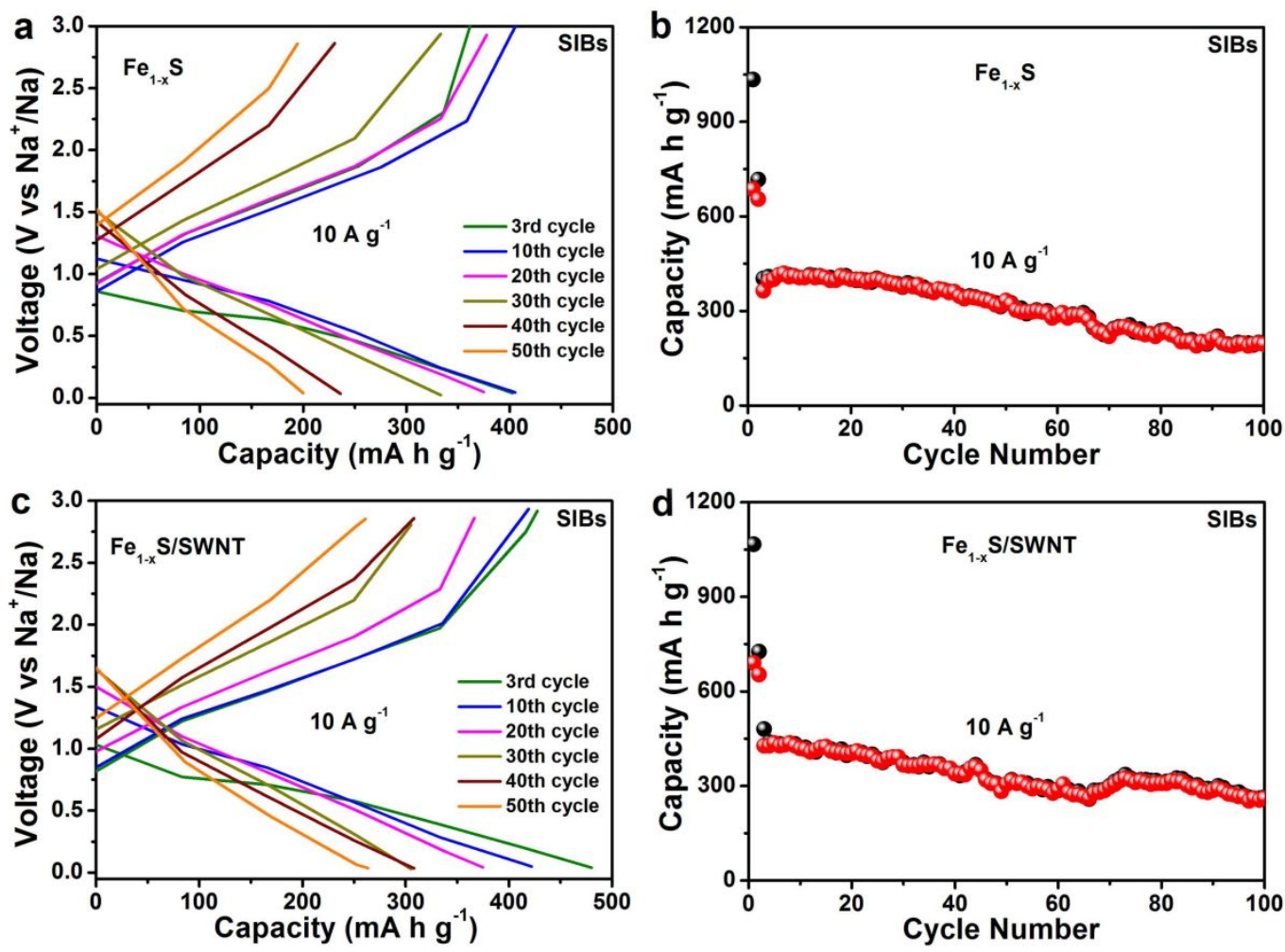

Figure S15. (a, c) Selected discharge/charge profiles, and (b, d) cycling performance of (a-b) $\mathrm{Fe}_{1-\mathrm{x}} \mathrm{S}$ and (c-d) $\mathrm{Fe}_{1-\mathrm{x}} \mathrm{S} / \mathrm{SWNT}$ anodes for SIBs at a high rate of $10 \mathrm{~A} \mathrm{~g}^{-1}$. 
Table S1. Comparison of as-reported yolk-shell anodes for SIBs.

\begin{tabular}{|c|c|c|c|c|c|}
\hline $\begin{array}{c}\text { Yolk-shell } \\
\text { structured anodes }\end{array}$ & $\begin{array}{l}\text { Current } \\
\text { density } \\
\left(\mathrm{A} \mathrm{g}^{-1}\right)\end{array}$ & $\begin{array}{l}\text { Cycle } \\
\text { Number }\end{array}$ & $\begin{array}{l}\text { Capacity } \\
\left(\mathrm{mAh} \mathrm{g}^{-1}\right)\end{array}$ & $\begin{array}{l}\text { Rate capacity } \\
\qquad\left(\mathbf{m A h ~ \mathbf { A } ^ { - 1 }}\right. \\
\left./ \mathrm{A} \mathrm{g}^{-1}\right)\end{array}$ & Ref \\
\hline $\mathrm{MoSe}_{2}$ & 0.2 & 50 & 433 & $345 / 1.5$ & $\mathrm{~S} 2$ \\
\hline SnS-MoS ${ }_{2}$ & 0.5 & 100 & 396 & 238/7.0 & $\mathrm{S} 3$ \\
\hline $\mathrm{Co}_{9} \mathrm{~S}_{8} / \mathrm{MoS}_{2}$ & $0.3 / 2$ & $100 / 1200$ & $476 / 300$ & $403 / 2$ & S4 \\
\hline FeS@C & 0.1 & 300 & 488 & $452 / 2.5$ & S5 \\
\hline $\mathrm{SnO}_{2} @ \mathrm{C}$ & 0.05 & 50 & 401 & $190 / 0.8$ & S6 \\
\hline $\mathrm{Sn}_{4} \mathrm{P}_{3} @ \mathrm{C}$ & $0.1 / 1.5$ & $50 / 400$ & $515 / 360$ & $421 / 3$ & S7 \\
\hline $\mathrm{Sn} @ \mathrm{C}$ & $0.1 / 1$ & $50 / 1000$ & $400 / 200$ & $150 / 5$ & $\mathrm{~S} 8$ \\
\hline Sb@Ti-O-P & 0.5 & 200 & 760 & $360 / 10$ & S9 \\
\hline $\mathrm{Sn}_{4} \mathrm{P}_{3} @ \mathrm{C}$ & $0.1 / 2$ & $50 / 500$ & $701 / 368$ & $508 / 2$ & S10 \\
\hline $\mathrm{FeS}_{2} @ \mathrm{C}$ & $0.1 / 2$ & $100 / 800$ & $511 / 330$ & $403 / 5$ & S11 \\
\hline $\mathrm{Sb} @ \mathrm{C}$ & 1 & 200 & 280 & $279 / 2$ & S12 \\
\hline $\mathrm{Sb} @ \mathrm{C}$ & 0.5 & 200 & 422 & $315 / 5$ & S13 \\
\hline $\mathrm{Co}_{3} \mathrm{O}_{4} @ \mathrm{C}$ & 1 & 200 & 240 & $269 / 2$ & S14 \\
\hline CoS@c & 0.2 & 100 & 532 & $190 / 20$ & S15 \\
\hline $\mathrm{Fe}_{1-\mathrm{x}} \mathrm{S} @ \mathrm{NC}$ & 0.1 & 100 & 594 & $470 / 2$ & S16 \\
\hline $\mathrm{MoSe}_{2} @ \mathrm{C}$ & 3 & 1000 & 378 & $308 / 10$ & S17 \\
\hline $\mathrm{Fe}_{3} \mathrm{~N} @ \mathrm{C}$ & 0.4 & 300 & 280 & $248 / 2$ & S18 \\
\hline $\mathrm{Co}_{2} \mathrm{P} / \mathrm{Sn} @ \mathrm{NC}$ & 5 & 10000 & 168 & $147 / 10$ & S19 \\
\hline $\mathrm{Ni}_{2} \mathrm{P} @ \mathrm{C}$ & 0.1 & 300 & 291 & $203 / 1$ & S20 \\
\hline $\mathrm{Co}_{9} \mathrm{~S}_{8} @ \mathrm{C}$ & 0.1 & 50 & $\sim 500$ & $100 / 10$ & $\mathrm{~S} 21$ \\
\hline $\mathrm{FeS}_{2} @ \mathrm{NC}$ & 5 & 1000 & 375 & $307 / 10$ & S22 \\
\hline $\mathbf{Y}-\mathbf{S}$ & 0.1 & 100 & 710 & $414 / 10$ & This \\
\hline $\mathrm{Fe}_{1-\mathrm{x}} \mathrm{S} / \mathrm{SWNT} @ \mathrm{C}$ & 2 & 8700 & 172 & $317 / 20$ & work \\
\hline
\end{tabular}




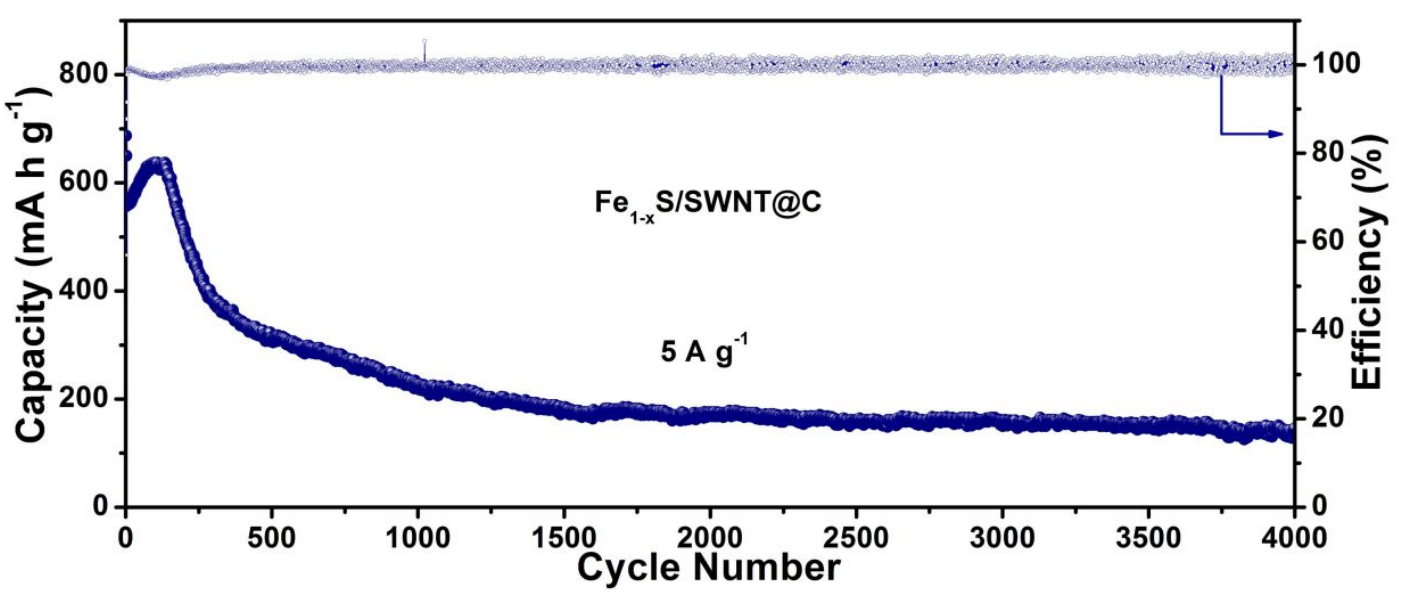

Figure S16. Long-term cycling stability of $\mathrm{Fe}_{1-\mathrm{x}} \mathrm{S} / \mathrm{SWNT} @ \mathrm{C}$ at a large current density of $5 \mathrm{~A} \mathrm{~g}^{-1}$ for SIBs, which delivered capacities of 312, 224, 171, and $146 \mathrm{~mA}$ $\mathrm{h} \mathrm{g}^{-1}$ after 500, 1000, 2000, and 4000 cycles, respectively. 
Table S2. Comparison of as-reported iron sulfide-based anodes for SIBs and PIBs.

\begin{tabular}{|c|c|c|c|c|c|c|c|}
\hline Anode Materials & $\begin{array}{l}\text { Current } \\
\text { density } \\
\left(\mathrm{A} \mathrm{g} \mathrm{g}^{-1}\right)\end{array}$ & $\begin{array}{c}\text { Cycle } \\
\text { Number }\end{array}$ & $\begin{array}{l}\text { Capacity } \\
\left(\mathrm{mAh} \mathrm{g}^{-1}\right)\end{array}$ & $\begin{array}{l}\text { Rate capability } \\
\qquad\left(\mathrm{mAh} \mathrm{g}^{-1}\right. \\
\left./ \mathrm{A} \mathrm{g}^{-1}\right)\end{array}$ & $\begin{array}{l}\text { Cut-off } \\
\text { voltage }\end{array}$ & $\begin{array}{l}\text { Kinds of } \\
\text { batteries }\end{array}$ & Ref \\
\hline $\mathrm{FeS}_{2}$ particles & 0.2 & 800 & 524 & $323 / 5$ & $0.5-3.0$ & SIBs & $\mathrm{S} 23$ \\
\hline Greigite $\mathrm{Fe}_{3} \mathrm{~S}_{4}$ & $5 / 20$ & $1000 / 3500$ & $435 / 275$ & $233 / 40$ & $0.5-3.0$ & SIBs & $\mathrm{S} 24$ \\
\hline FeS@C & $0.09 / 0.7$ & $50 / 200$ & $430 / 140$ & $280 / 4.5$ & $0.5-3.0$ & SIBs & S25 \\
\hline $\mathrm{FeS}_{2} / \mathrm{rGO}$ aerogel & 0.9 & 800 & 181 & $195 / 4.5$ & $0.8-2.8$ & SIBs & S26 \\
\hline $\mathrm{FeS}_{2} / \mathrm{CNT}$ network & $0.1 / 1$ & $400 / 1800$ & $394 / 309$ & - & $0.8-3.0$ & SIBs & $\mathrm{S} 27$ \\
\hline Y-S FeS $2 @ N C$ & $0.2 / 5$ & $200 / 1000$ & $551 / 375$ & $307 / 10$ & $0.5-3.0$ & SIBs & S22 \\
\hline Y-SFeS@C & 0.1 & 300 & 488 & $452 / 2.5$ & $0.05-3.0$ & SIBs & S5 \\
\hline FeS@rGO & 0.15 & 50 & 547 & $367 / 5$ & $0.001-3$ & SIBs & S28 \\
\hline $\mathrm{C} / \mathrm{FeS}$ microsphere & 1 & 200 & 300 & $65 / 5$ & $0.01-3.0$ & SIBs & S29 \\
\hline $\mathrm{FeS}_{\mathrm{x}} @ \mathrm{C}$ & 0.1 & 636 & 100 & $403 / 2$ & $0-2.5$ & SIBs & $\mathrm{S} 30$ \\
\hline $\mathrm{Fe}_{1-\mathrm{x}} \mathrm{S}$ & $0.1 / 1$ & $200 / 2000$ & $536 / 475$ & $300 / 10$ & $0.005-3$ & SIBs & $\mathrm{S} 31$ \\
\hline $\mathrm{Fe}_{1-\mathrm{x}} \mathrm{S} @ \mathrm{CNT}$ & 0.5 & 200 & 449 & $326 / 8$ & $0.01-2.3$ & SIBs & $\mathrm{S} 32$ \\
\hline Ultrafine $\mathrm{Fe}_{7} \mathrm{~S}_{8} @ \mathrm{C}$ & 0.18 & 1000 & 447 & $552 / 2.7$ & $0.08-3.0$ & SIBs & S33 \\
\hline $\mathrm{FeS}_{2} @ \mathrm{rGO}$ & 0.1 & 100 & 609 & $344 / 10$ & $0.01-2.3$ & SIBs & S34 \\
\hline Y-S FeS ${ }_{2} @ \mathrm{C}$ & $0.1 / 2$ & $100 / 800$ & $511 / 330$ & $403 / 5$ & $0.2-2.0$ & SIBs & S11 \\
\hline $\mathrm{Fe}_{7} \mathrm{~S}_{8} @ \mathrm{C}-\mathrm{G}$ & 0.5 & 150 & 449 & $306 / 2$ & $0.01-3.0$ & SIBs & S35 \\
\hline G/FeS@C aerogel & 0.1 & 80 & 632 & $363 / 1$ & $0.01-3.0$ & SIBs & $\mathrm{S} 36$ \\
\hline $\mathrm{Fe}_{1-\mathrm{x}} \mathrm{S} @ \mathrm{NC} @ \mathrm{G}$ & 0.2 & 500 & 385 & $243 / 10$ & $0-2.5$ & SIBs & S37 \\
\hline Y-S Fe ${ }_{1-x} \mathrm{~S} @ N C$ & 0.1 & 100 & 594 & $470 / 2$ & $0.01-2.5$ & SIBs & S16 \\
\hline $\mathrm{Fe}_{1-\mathrm{x}} \mathrm{S} @ \mathrm{PC} / \mathrm{G}$ & 0.1 & 100 & 573 & $240 / 5$ & $0.01-2.3$ & SIBs & $\mathrm{S} 1$ \\
\hline $\mathrm{FeS}_{2} @ \mathrm{rGO}$ & 0.05 & 50 & 264 & $151 / 0.5$ & $0.01-3.0$ & PIBs & S38 \\
\hline $\mathrm{G} @ \mathrm{Y}-\mathrm{S} \mathrm{FeS}_{2} @ \mathrm{C}$ & 0.3 & 1000 & 270 & $166 / 15$ & $0.05-2.8$ & PIBs & S39 \\
\hline $\mathrm{FeS}_{2} @ \mathrm{C}$ & 1 & 150 & 295 & $182 / 10$ & $0.01-3$ & PIBs & $\mathrm{S} 40$ \\
\hline $\mathrm{FeS}_{2} @ \mathrm{G} @ \mathrm{CNF}$ & 0.2 & 100 & 205 & $171 / 1$ & $0.01-3$ & PIBs & S41 \\
\hline $\mathbf{Y}-\mathbf{S}$ & $0.1 / 2$ & $100 / 8700$ & $710 / 172$ & $317 / 20$ & \multirow{2}{*}{$0.05-2.8$} & SIBs & This \\
\hline $\mathrm{Fe}_{1-\mathrm{x}} \mathrm{S} / \mathrm{SWNT} @ \mathrm{C}$ & 0.1 & 50 & 472 & $172 / 20$ & & PIBs & work \\
\hline
\end{tabular}




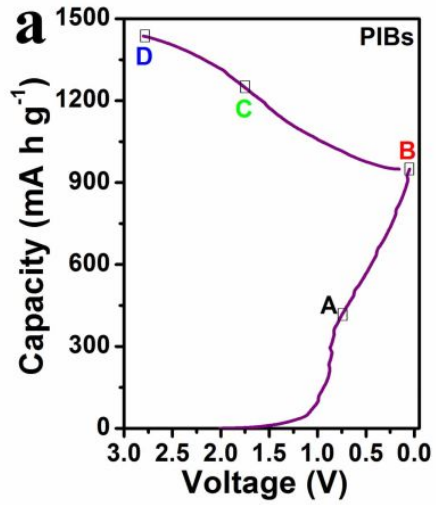

C

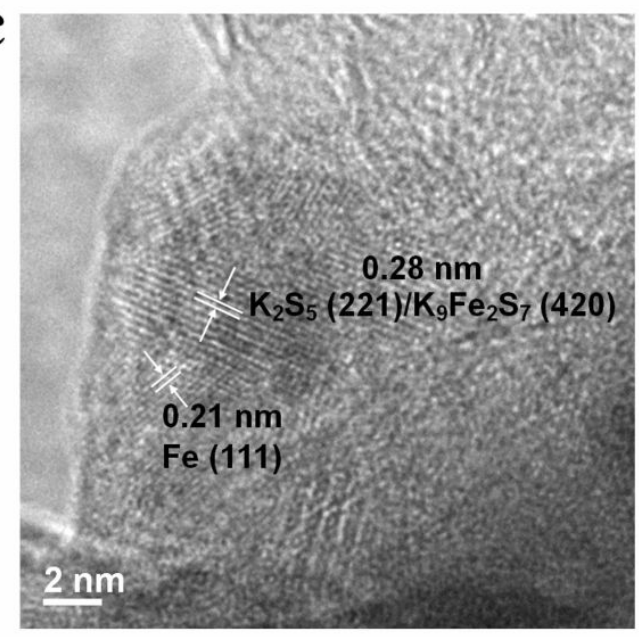

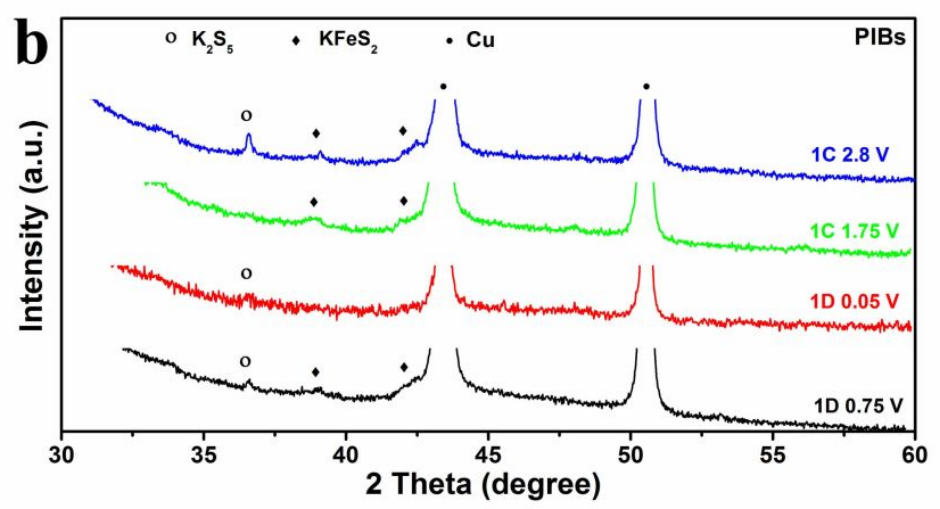

d

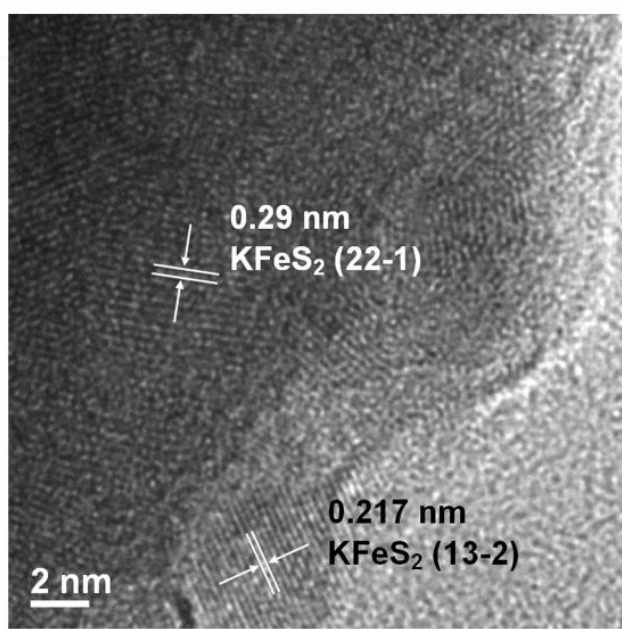

Figure S17. (a) Initial discharge/charge curves at $50 \mathrm{~mA} \mathrm{~g}^{-1}$ for PIBs, and (b) ex-situ XRD patterns of $\mathrm{Fe}_{1-\mathrm{x}} \mathrm{S} / \mathrm{SWNT} @ \mathrm{C}$ electrode under various states. HRTEM images of $\mathrm{Fe}_{1-\mathrm{x}} \mathrm{S} / \mathrm{SWNT} @ \mathrm{C}$ electrode after (c) initial discharge to $0.05 \mathrm{~V}$, and (d) initial charge to $2.8 \mathrm{~V}$ for PIBs. In Figure S15b, the diffraction peaks of $\mathrm{KFeS}_{2}$ phase were detected at $1 \mathrm{D} 0.75 \mathrm{~V}, 1 \mathrm{C} 1.75 \mathrm{~V}$, and $1 \mathrm{C} 2.8 \mathrm{~V}$, implying the reversible formation of K-rich phase and potassium intercalation/deintercalation process in this phase. After initial discharging to $0.05 \mathrm{~V}$, the ex-HRTEM image in Figure S15c disclosed the occurrence of conversion reaction to generate $\mathrm{Fe}$ and $\mathrm{K}_{2} \mathrm{~S}_{5}$. Figure $\mathrm{S} 15 \mathrm{~d}$ clarified the existence of $\mathrm{KFeS}_{2}$ phase when first charged to $2.8 \mathrm{~V}$. 

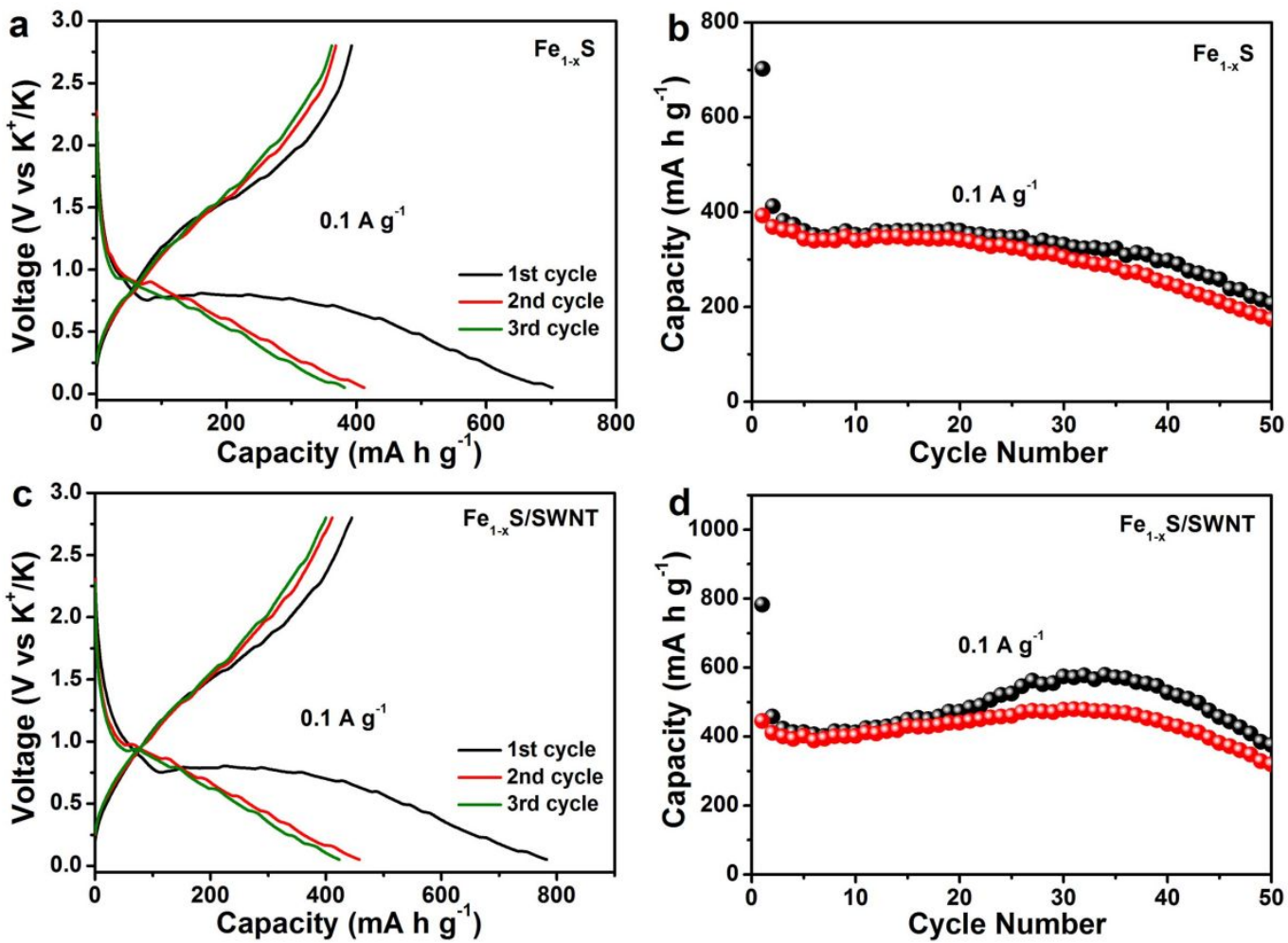

Figure S18. (a, c) Discharge/charge curves in the first three cycles, and (b, d) cycling performance of (a-b) $\mathrm{Fe}_{1-\mathrm{x}} \mathrm{S}$ and (c-d) $\mathrm{Fe}_{1-\mathrm{x}} \mathrm{S} / \mathrm{SWNT}$ anodes for PIBs at $0.1 \mathrm{~A} \mathrm{~g}^{-1}$. As can be seen, bare $\mathrm{Fe}_{1-\mathrm{x}} \mathrm{S}$ anode suffered from fast capacity decay and only kept a low capacity of $174 \mathrm{~mA} \mathrm{~h} \mathrm{~g}{ }^{-1}$ after 50 cycles. The SWNT layer enhanced the capacity retention of $\mathrm{Fe}_{1-\mathrm{x}} \mathrm{S}$, leading to a capacity $320 \mathrm{~mA} \mathrm{~h} \mathrm{~g}{ }^{-1}$ after 50 cycles for $\mathrm{Fe}_{1-\mathrm{x}} \mathrm{S} / \mathrm{SWNT}$ anode. 

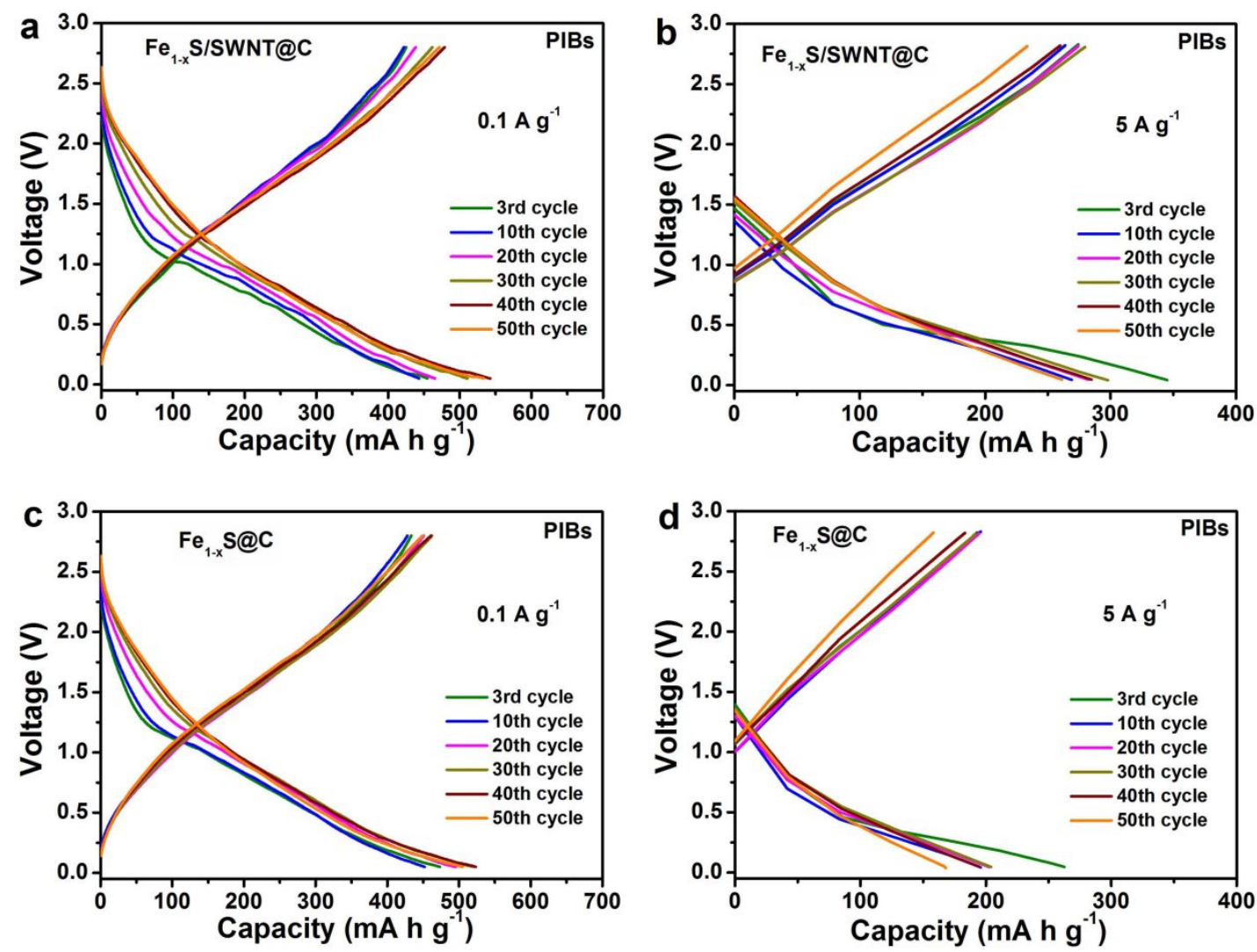

Figure S19. Selected discharge/charge profiles of (a-b) Fe $\mathrm{F}_{1-\mathrm{x}} \mathrm{S} / \mathrm{SWNT} @ \mathrm{C}$ and (c-d) $\mathrm{Fe}_{1-\mathrm{x}} \mathrm{S} @ \mathrm{C}$ anodes at $(\mathrm{a}, \mathrm{c}) 0.1$ and $(\mathrm{b}, \mathrm{d}) 5 \mathrm{~A} \mathrm{~g}^{-1}$ for PIBs. It can be seen that these two anodes showed similar cycling stability at $0.1 \mathrm{~A} \mathrm{~g}^{-1}$, consistent with the sodium storage behavior at low current density. However, at a high rate of $5 \mathrm{~A} \mathrm{~g}^{-1}$, the discharge and charge profiles of $\mathrm{Fe}_{1-\mathrm{x}} \mathrm{S} / \mathrm{SWNT} @ \mathrm{C}$ anode delivered higher capacities and lower overpotentials than that of $\mathrm{Fe}_{1-\mathrm{x}} \mathrm{S} @ \mathrm{C}$ anode. 


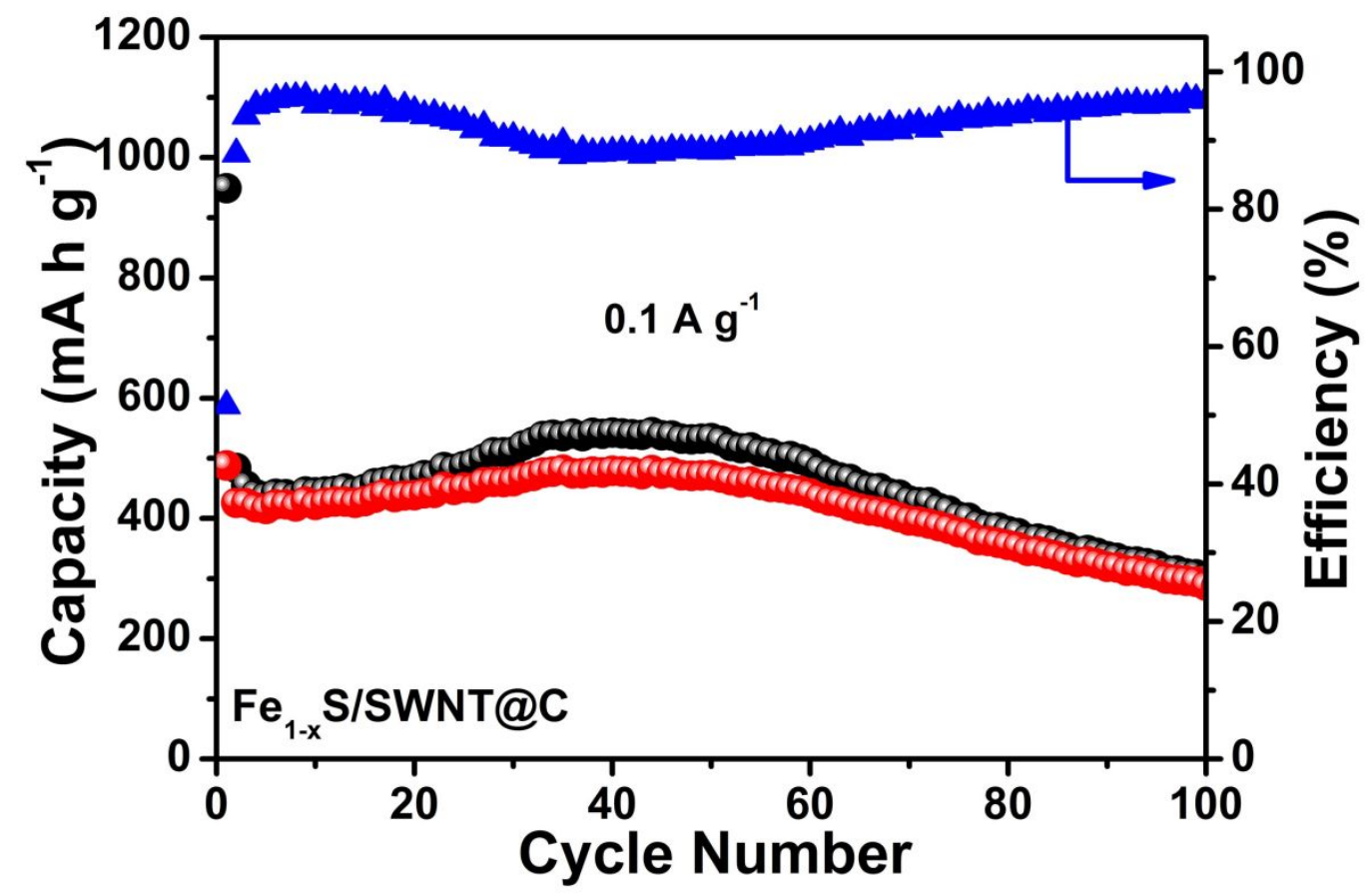

Figure S20. Cycling performance and related columbic efficiencies of $\mathrm{Fe}_{1-\mathrm{x}} \mathrm{S} / \mathrm{SWNT} @ \mathrm{C}$ anode at $0.1 \mathrm{~A} \mathrm{~g}^{-1}$ up to 100 cycles for PIBs. This anode underwent an activation process in the initial cycles with a maximum reversible capacity of 480 $\mathrm{mA} \mathrm{h} \mathrm{g}{ }^{-1}$ obtained at $40^{\text {th }}$ cycle at $0.1 \mathrm{~A} \mathrm{~g}^{-1}$. This process was accompanied with the decrease of $\mathrm{CE}$, probably owing to the exposure of new fresh active materials with increased side reaction and more electrolyte consumption. Then, it experienced capacity decay since from $50^{\text {th }}$ cycle. The possible reason could be due to the insufficient charge transfer between yolk and shell, and the dissolution of potassium polysulfides during cycles. Even though, this anode still remained a discharge capacity of $301 \mathrm{~mA} \mathrm{~h} \mathrm{~g}^{-1}$ after 100 cycles at $0.1 \mathrm{~A} \mathrm{~g}^{-1}$. 


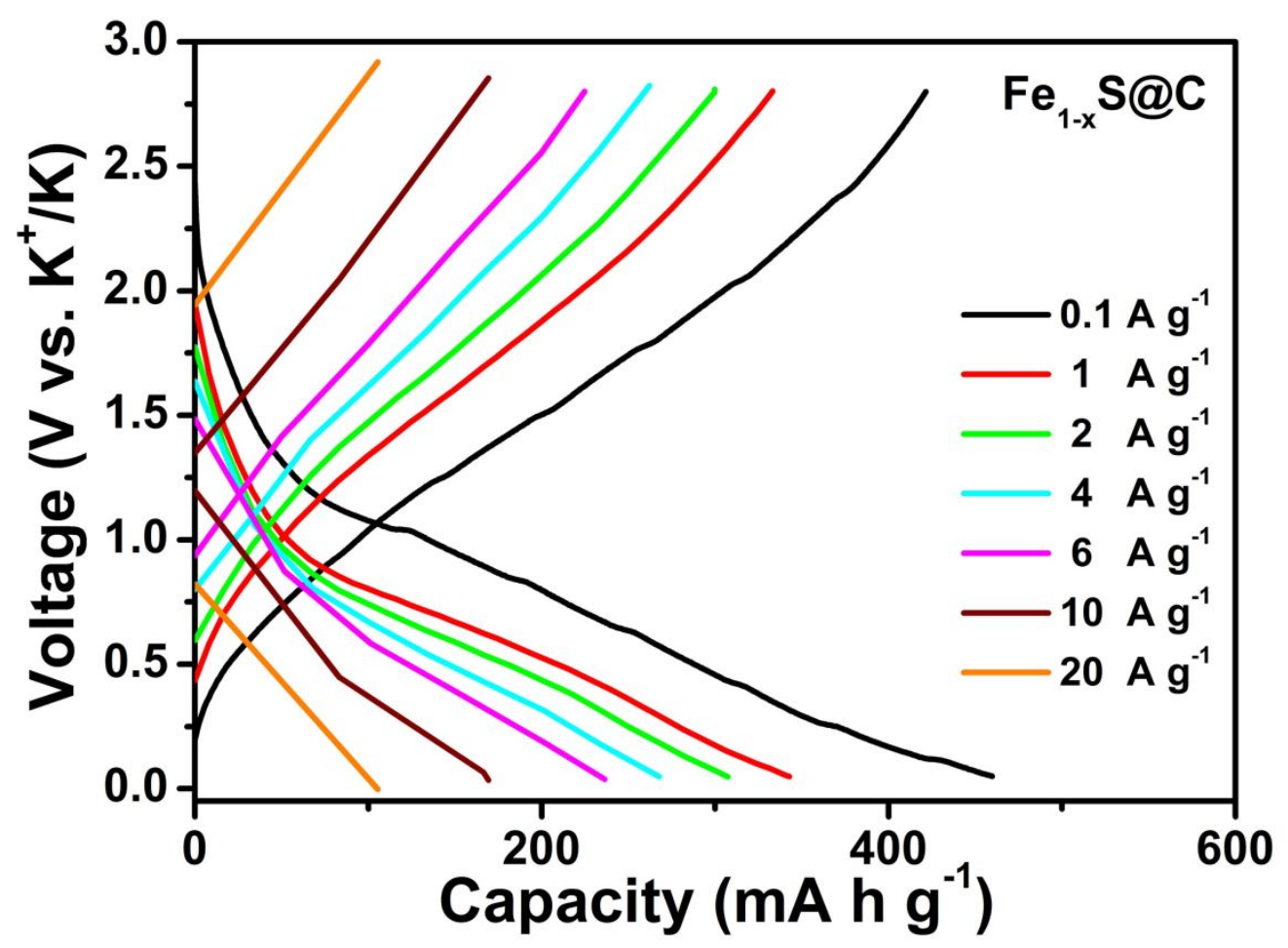

Figure S21. Typical discharge/charge profiles of $\mathrm{Fe}_{1-\mathrm{x}} \mathrm{S} @ \mathrm{C}$ anode under various rates from 0.1 to $20 \mathrm{~A} \mathrm{~g}^{-1}$ for PIBs. Compared with $\mathrm{Fe}_{1-\mathrm{x}} \mathrm{S} / \mathrm{SWNT} @ \mathrm{C}$ anode, the discharge and charge profiles of $\mathrm{Fe}_{1-\mathrm{x}} \mathrm{S} @ \mathrm{C}$ showed lower capacities and larger overpotentials at high current densities. 
Table S3. Comparison of as-reported anode materials for PIBs.

\begin{tabular}{|c|c|c|c|c|c|}
\hline Anode Materials & $\begin{array}{l}\text { Current } \\
\text { density } \\
\left(\mathrm{A} \mathrm{g}^{-1}\right)\end{array}$ & $\begin{array}{c}\text { Cycle } \\
\text { Number }\end{array}$ & $\begin{array}{l}\text { Capacity } \\
\left(\mathrm{mAh} \mathrm{g}^{-1}\right)\end{array}$ & $\begin{array}{l}\text { Rate capacity } \\
\qquad\left(\mathrm{mAh} \mathrm{g}^{-1}\right. \\
\left./ \mathrm{A} \mathrm{g}^{-1}\right)\end{array}$ & Ref \\
\hline Graphite & 0.14 & 50 & 100 & $80 / 0.28$ & S42 \\
\hline N-doped carbon monolith & 0.02 & 60 & 351 & $178 / 5$ & S43 \\
\hline $\mathrm{N}, \mathrm{O}$-doped hard carbon & 0.05 & 100 & 230 & $118 / 3$ & S44 \\
\hline S, N-doped carbon shell & 0.1 & 400 & 200 & $64 / 4$ & S45 \\
\hline $\mathrm{SnS}_{2}$-graphene & 0.025 & 30 & 250 & $120 / 2$ & $\mathrm{~S} 46$ \\
\hline $\mathrm{VS}_{2}$ nanosheets & 0.1 & 60 & 410 & $100 / 2$ & S47 \\
\hline $\mathrm{Co}_{3} \mathrm{O}_{4}-\mathrm{Fe}_{2} \mathrm{O}_{3} / \mathrm{C}$ & 0.05 & 50 & 220 & - & $\mathrm{S} 48$ \\
\hline $\mathrm{Sb}_{2} \mathrm{~S}_{3} / \mathrm{S}, \mathrm{N}-\mathrm{G}$ & 0.05 & 100 & 480 & $340 / 1$ & S49 \\
\hline $\mathrm{MoS}_{2}$ nanorose & 0.1 & 100 & 380 & $178 / 0.5$ & S50 \\
\hline CoS-graphene & 0.5 & 100 & 310 & $230 / 4$ & S51 \\
\hline $\mathrm{Sn}_{4} \mathrm{P}_{3} / \mathrm{C}$ & 0.05 & 50 & 307 & $222 / 1$ & S52 \\
\hline $\mathrm{ReS}_{2} / \mathrm{N}$-doped CNF & 0.05 & 100 & 253 & - & S53 \\
\hline $\mathrm{K}_{0.6} \mathrm{Mn}_{1} \mathrm{~F}_{2.7}$ hollow cube & 0.05 & 100 & 138 & $78 / 1$ & S54 \\
\hline $\mathrm{NCNF@CoSe} 2$ & 0.2 & 100 & 253 & $196 / 2$ & S55 \\
\hline $\mathrm{MoSe}_{2} @ \mathrm{C}$ & 0.2 & 100 & 322 & $224 / 2$ & S56 \\
\hline $\mathrm{CoP} / \mathrm{N}, \mathrm{P}$-carbon sheets & 0.1 & 1000 & 127 & $54 / 2$ & S57 \\
\hline $\mathrm{MoS}_{2} / \mathrm{N}-\mathrm{C}$ tube & 0.05 & 50 & 330 & $131 / 2$ & S58 \\
\hline $\mathrm{MoSe}_{2} @ \mathrm{~N}-\mathrm{C}$ & 0.1 & 300 & 258 & $197 / 1$ & S59 \\
\hline $\mathrm{MoSe}_{2} / \mathrm{Mxene} @ \mathrm{C}$ & 0.2 & 100 & 355 & $183 / 10$ & S60 \\
\hline Y-SFeP@C & 0.1 & 300 & 205 & $37 / 2$ & S61 \\
\hline $\mathrm{SnS}_{2} @ \mathrm{C} @ \mathrm{rGO}$ & 0.2 & 500 & 298 & $288 / 0.5$ & S62 \\
\hline $\mathrm{FeS}_{2} @ \mathrm{C}$ & 1 & 150 & 295 & $182 / 10$ & S40 \\
\hline $\mathrm{Bi}_{1.11} \mathrm{Sb}_{0.89} \mathrm{~S}_{3}$ & 0.5 & 1000 & 353 & $300 / 1$ & S63 \\
\hline 3D Fe $\mathrm{x}_{\mathrm{x}} \mathrm{O} @ \mathrm{~N}-\mathrm{G}$ & 0.05 & 100 & 423 & $175 / 5$ & S64 \\
\hline $\begin{array}{c}\mathrm{Y}-\mathrm{S} \\
\mathrm{Fe}_{1-\mathrm{x}} \mathrm{S} / \mathrm{SWNT} @ \mathrm{C}\end{array}$ & 0.1 & 50 & 472 & $\begin{array}{l}236 / 10 \\
172 / 20\end{array}$ & $\begin{array}{l}\text { This } \\
\text { work }\end{array}$ \\
\hline
\end{tabular}



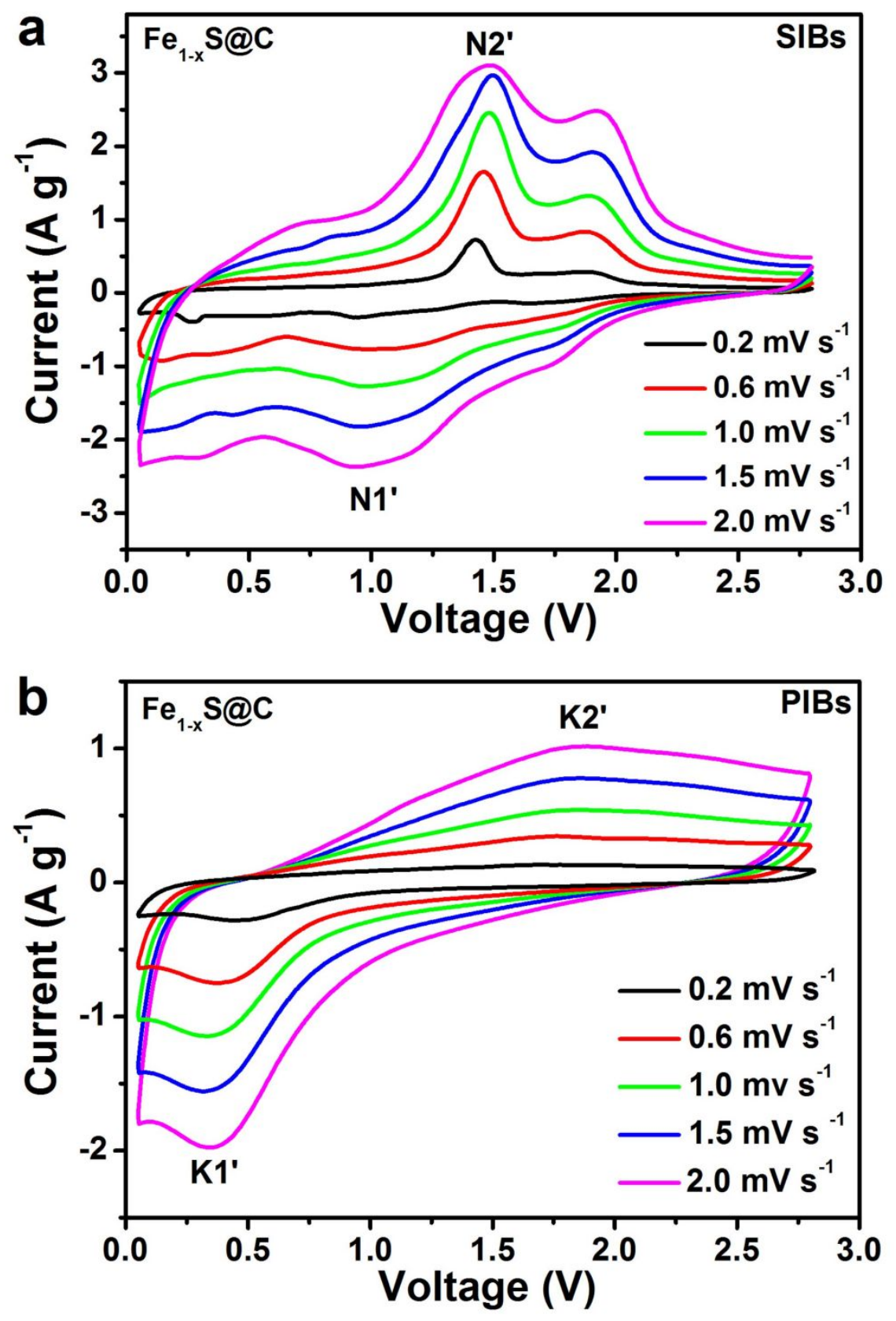

Figure S22. CV profiles of $\mathrm{Fe}_{1-\mathrm{x}} \mathrm{S} @ \mathrm{C}$ anode under various scan rates from 0.2 to 2.0 $\mathrm{mV} \mathrm{s}^{-1}$ for (a) SIBs and (b) PIBs. 

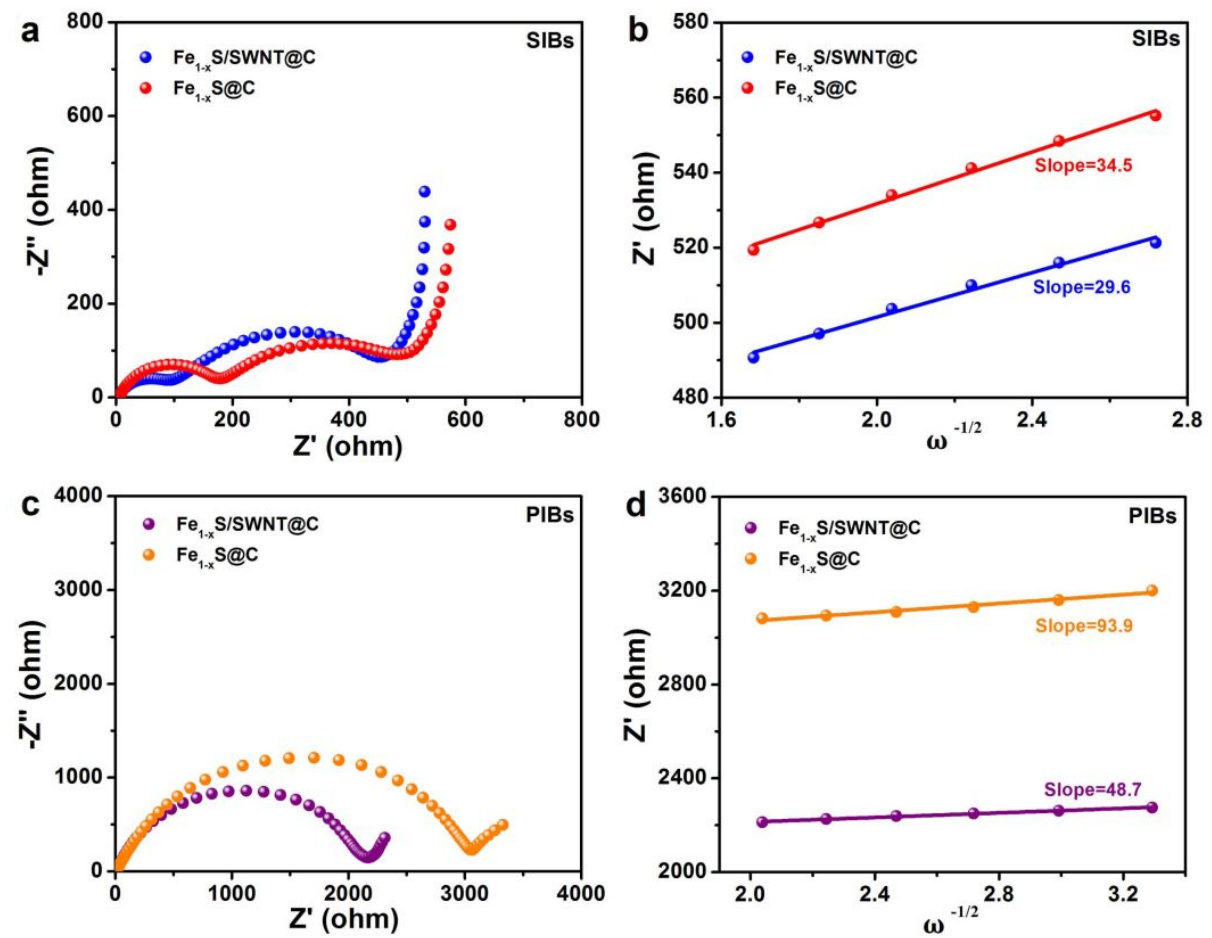

Figure S23. (a, c) EIS profiles, and (b, d) linear relationship between $Z_{\text {real }}$ and $\omega^{-1 / 2}$ at low frequencies of $\mathrm{Fe}_{1-\mathrm{x}} \mathrm{S} @ \mathrm{C}$ and $\mathrm{Fe}_{1-\mathrm{x}} \mathrm{S} / \mathrm{SWNT} @ \mathrm{C}$ anodes after 3 cycles at the fully discharged state for (a-b) SIBs and (c-d) PIBs. As can be seen, $\mathrm{Fe}_{1-\mathrm{x}} \mathrm{S} / \mathrm{SWNT}$ @C exhibited smaller semicircle diameter than $\mathrm{Fe}_{1-\mathrm{x}} \mathrm{S} @ \mathrm{C}$ in the high-medium frequency region for both SIBs and PIBs. It implied that SWNT channels can effectively improve the electrical conductivity and reduce the charge-transfer and contact resistances of yolk shell structure. The ion diffusion coefficient calculated based on the EIS profiles demonstrated that SWNT channels can effectively enhance the ion diffusion kinetics of Y-S structure.

Calculation details: According to previous literatures, ${ }^{\mathrm{S} 65-67}$ the ion diffusion coefficient could be calculated based on the linear fitting results of EIS profiles in the low frequency and the following equation:

$$
\mathrm{D}=0.5 \mathrm{R}^{2} \mathrm{~T}^{2} / \mathrm{A}^{2} \mathrm{n}^{4} \mathrm{~F}^{4} \mathrm{C}^{2} \sigma^{2}
$$

In which $\mathrm{R}$ is the gas constant, $\mathrm{T}$ is the absolute temperature, $\mathrm{A}$ is the surface area of the electrode, $\mathrm{n}$ is the electron transfer number of per molecule, $\mathrm{C}$ is the concentration of sodium or potassium ions, and $\sigma$ is the Warburg factor. Figure $5 \mathrm{~b}$ and $5 \mathrm{~d}$ exhibit the linear fitting of $Z_{\text {real }}$ and $\omega^{-1 / 2}$, from which the slop $\sigma$ can be obtained. Therefore, the $\mathrm{Na}^{+}$diffusion coefficients of $\mathrm{Fe}_{1-\mathrm{x}} \mathrm{S} / \mathrm{SWNT} @ \mathrm{C}$ and $\mathrm{Fe}_{1-\mathrm{x}} \mathrm{S} @ \mathrm{C}$ were calculated to be $1.0 \times 10^{-11}$ and $7.4 \times 10^{-12} \mathrm{~cm}^{2} \mathrm{~s}^{-1}$, respectively. The $\mathrm{D}_{\mathrm{k}+}$ values for $\mathrm{Fe}_{1-\mathrm{x}} \mathrm{S} / \mathrm{SWNT} @ \mathrm{C}$ and $\mathrm{Fe}_{1-\mathrm{x}} \mathrm{S} @ \mathrm{C}$ were $3.7 \times 10^{-12}$ and $1.0 \times 10^{-12} \mathrm{~cm}^{2} \mathrm{~s}^{-1}$, respectively. 

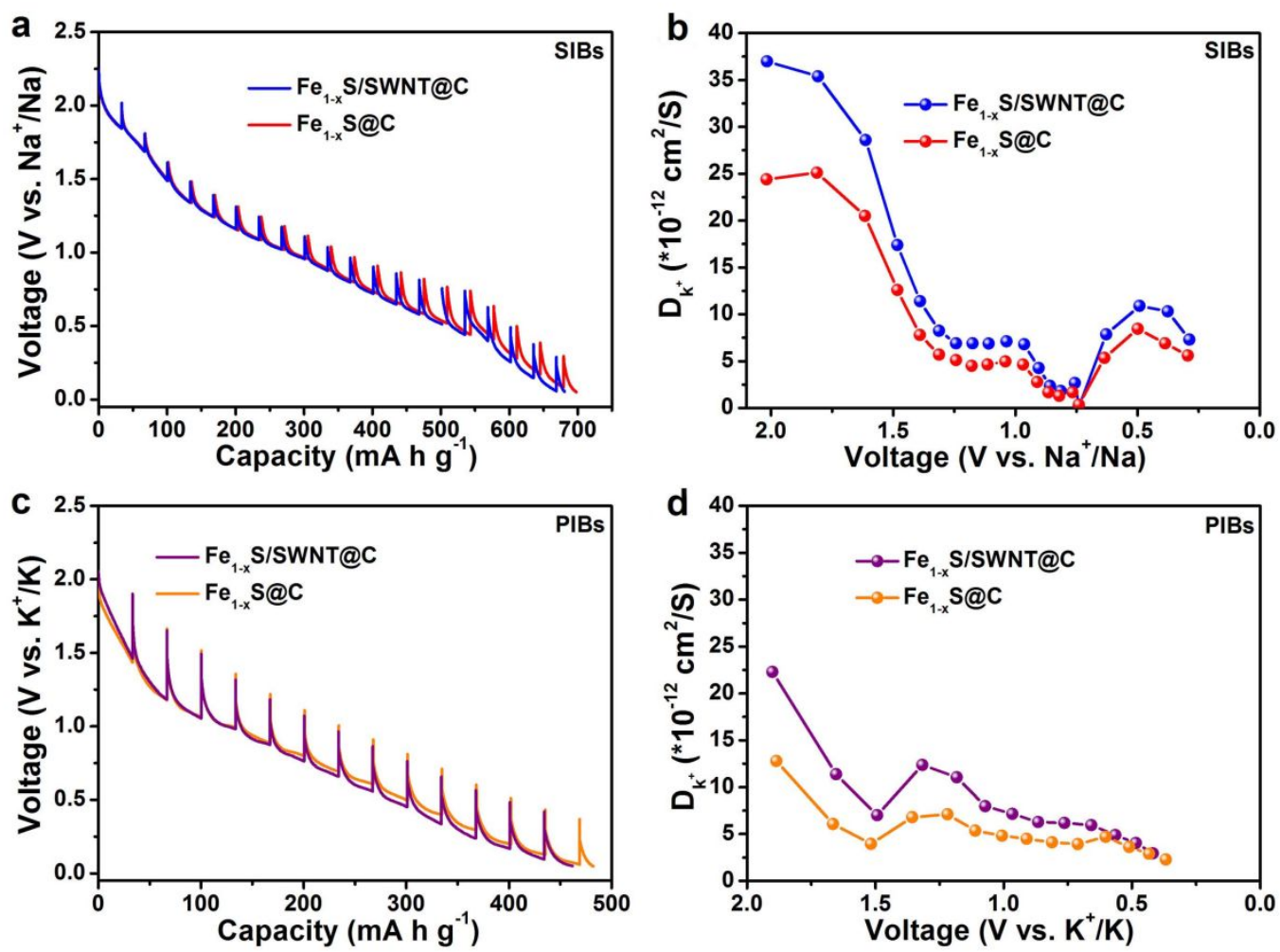

Figure S24. (a) GITT profiles of $\mathrm{Fe}_{1-\mathrm{x}} \mathrm{S} @ \mathrm{C}$ and $\mathrm{Fe}_{1-\mathrm{x}} \mathrm{S} / \mathrm{SWNT} @ \mathrm{C}$ electrodes during the sodiation process, and (b) the corresponding Na-ion diffusion coefficient as a function of the state of discharging process. (c) GITT profiles and (d) K-ion diffusion coefficients during potassiation process of $\mathrm{Fe}_{1-\mathrm{x}} \mathrm{S} @ \mathrm{C}$, and $\mathrm{Fe}_{1-\mathrm{x}} \mathrm{S} / \mathrm{SWNT} @ \mathrm{C}$ electrodes. Data was recorded with a series of current pulses at $100 \mathrm{~mA} \mathrm{~g}^{-1}$ for $20 \mathrm{~min}$ and $2 \mathrm{~h}$ relaxation at each interval.

Calculation details: The ion diffusion coefficient $(D i)$ can be quantitatively calculated based on the simplified Fick's second law (Equation (4)): ${ }^{\mathrm{S} 68-71}$

$D_{i}=\frac{4}{\pi \tau}\left(\frac{m_{B} V_{m}}{M_{B} A}\right)^{2}\left(\frac{\Delta E_{s}}{\Delta E_{\tau}}\right)^{2}$

Where $V_{m}, M_{B}$, and $m_{B}$ are the molar volume, the molecular weight, and the mass of the compound, respectively. $\mathrm{A}$ is the geometric area of the electrode. $\Delta E_{s}$ and $\Delta E_{\tau}$ are the voltage changes during the time period $\tau(20 \mathrm{~min})$ and after $2 \mathrm{~h}$ of open-circuit stand. Figure $\mathrm{x}$ shows the GITT curves of these two electrodes during sodiation and potassiation processes and the corresponding ion diffusion coefficient. As can be seen, the highest $\mathrm{D}_{\mathrm{Na}^{+}}$and $\mathrm{D}_{\mathrm{K}+}$ of $\mathrm{Fe}_{1-\mathrm{x}} \mathrm{S} / \mathrm{SWNT} @ \mathrm{C}$ were 1.5 and 1.7 times higher than that of $\mathrm{Fe}_{1-\mathrm{x}} \mathrm{S} @ \mathrm{C}$, respectively. It implies that the construction of SWNT channels within yolk-shell structure can facilitate the fast ion transportation. 
a
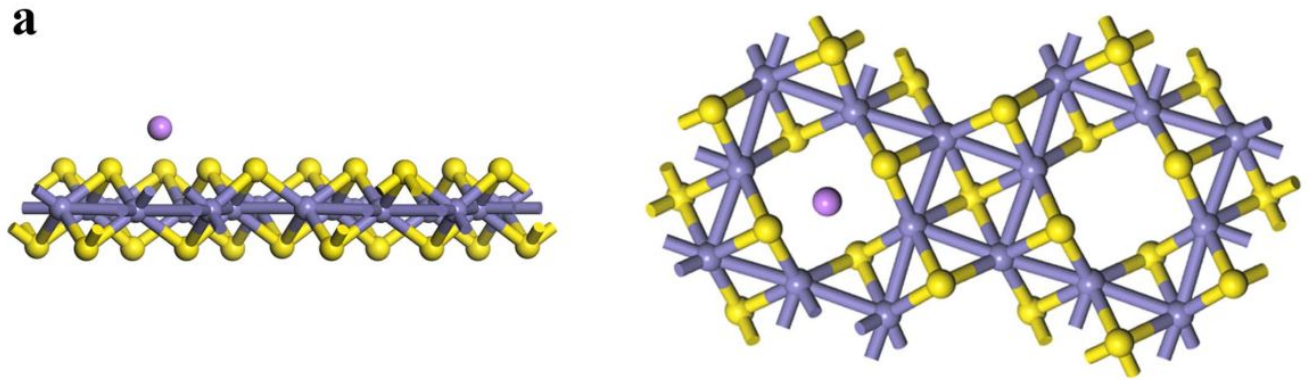

b
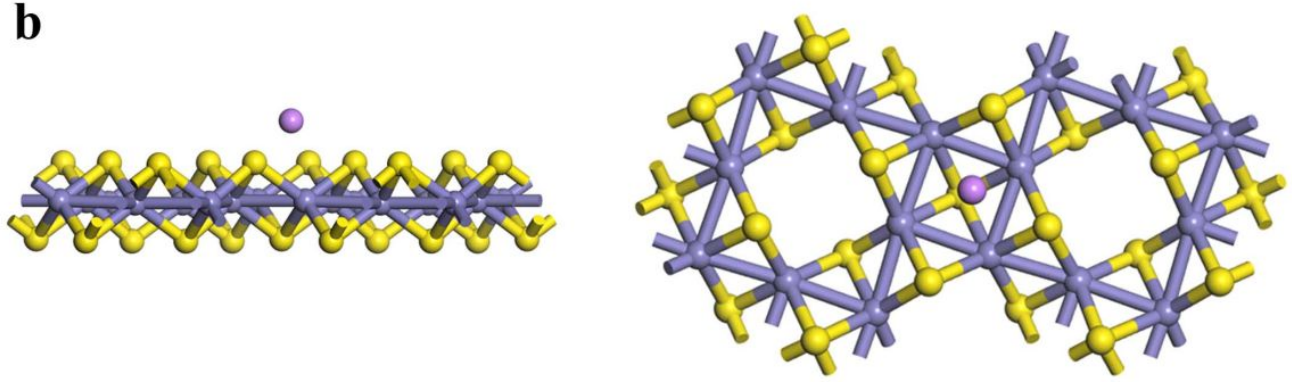

c
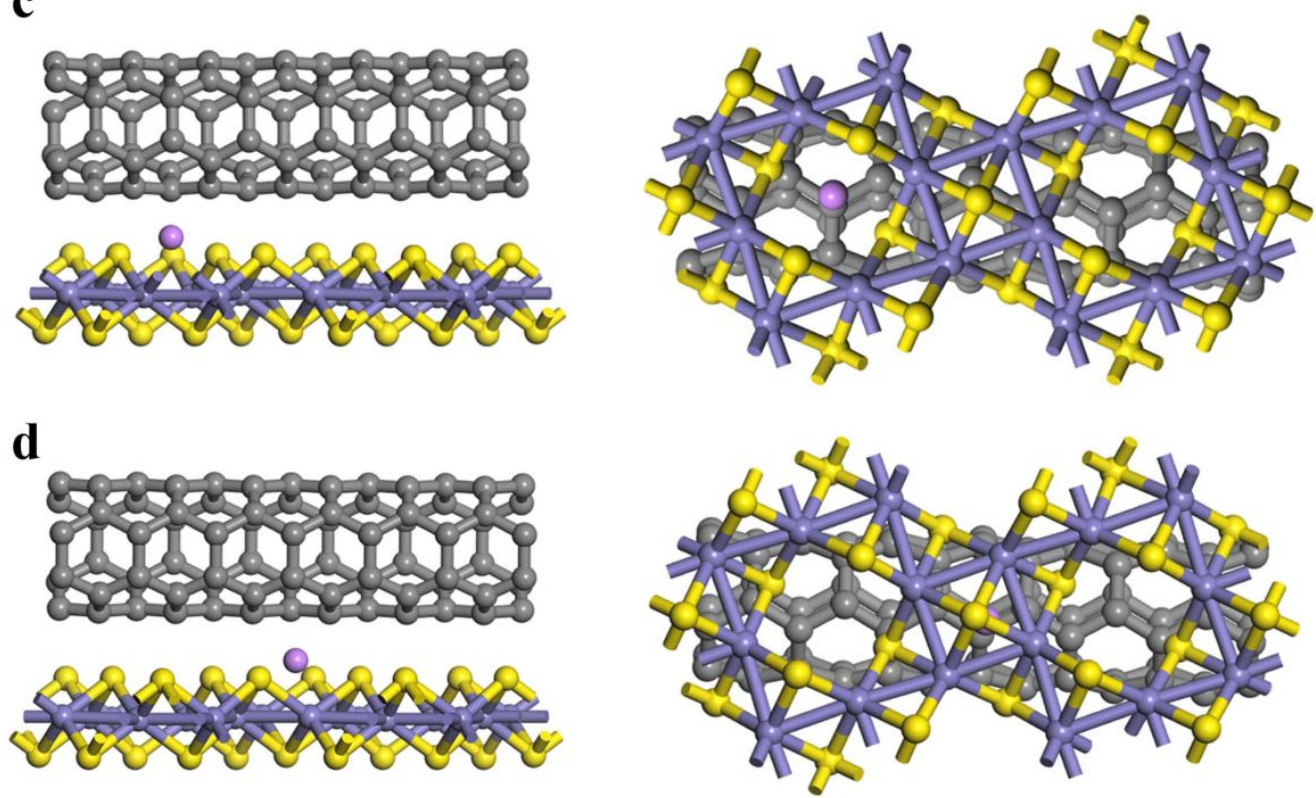

Figure S25. Side and top views of (a-b) $\mathrm{Fe}_{1-\mathrm{x}} \mathrm{S}$ (001) surface and (c-d) $\mathrm{Fe}_{1-\mathrm{x}} \mathrm{S} / \mathrm{SWNT}$ interface under different adsorption sites of $(\mathrm{a}, \mathrm{c}) \mathrm{K} 1$ atom and (b, d) K2 atom. Herein, the absorption energy $\left(E_{a d}\right)$ was calculated by the following Equation: $E_{a d}=E_{\text {total }}-\left(E_{a}+\right.$ $E_{K}$ ), where the $E_{\text {total }}$ is the total energy of $\mathrm{K}$ adsorbed in $\mathrm{Fe}_{1-\mathrm{x}} \mathrm{S}$ or interface. $\mathrm{E}_{\mathrm{a}}$ is the total energy of $\mathrm{Fe}_{1-\mathrm{x}} \mathrm{S}$ or interface, $\mathrm{E}_{\mathrm{k}}$ is the energy of $\mathrm{K}$ atoms. 
Table S4. The adsorption energy $\left(\mathrm{E}_{\mathrm{ad}}\right)$ of different $\mathrm{K}$ atom adsorption sites in the $\mathrm{Fe}_{1-\mathrm{x}} \mathrm{S}$ surface and $\mathrm{Fe}_{1-\mathrm{x}} \mathrm{S} / \mathrm{SWNT}$ heterointerface.

\begin{tabular}{llr}
\hline Structure & Adsorption site & $\mathrm{E}_{\mathrm{ad}}(\mathrm{eV})$ \\
\hline $\mathrm{Fe}_{1-\mathrm{x}} \mathrm{S}(001)$ & $\mathrm{K} 1$ & -0.75 \\
$\mathrm{Fe}_{1-\mathrm{x}} \mathrm{S}(001)$ & $\mathrm{K} 2$ & -1.32 \\
$\mathrm{Fe}_{1-\mathrm{x}} \mathrm{S} / \mathrm{SWNT}$ & $\mathrm{K} 1$ & -0.83 \\
$\mathrm{Fe}_{1-\mathrm{x}} \mathrm{S} / \mathrm{SWNT}$ & $\mathrm{K} 2$ & -1.42 \\
\hline
\end{tabular}



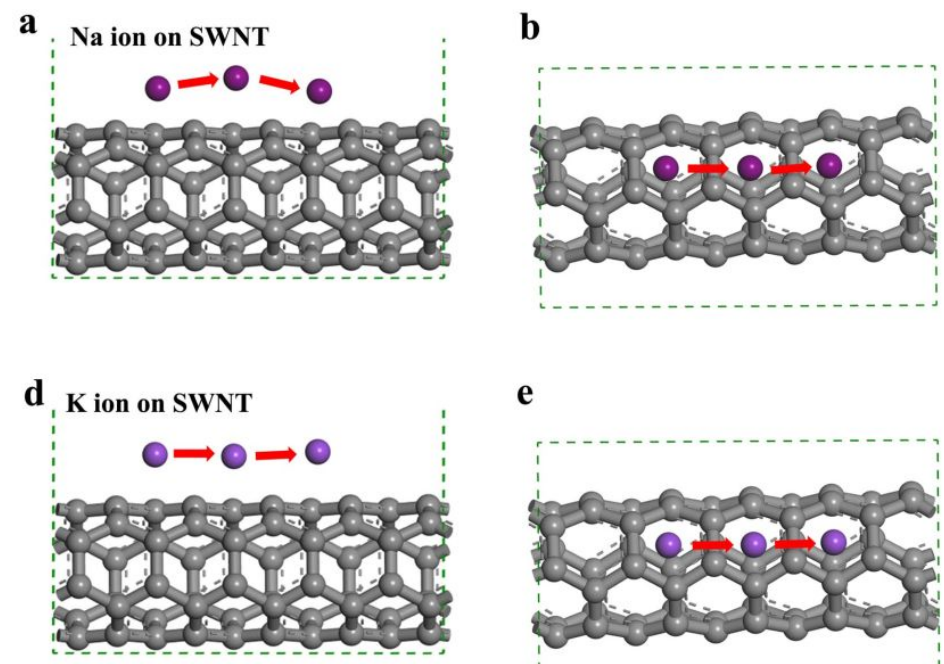

○Na OK ○

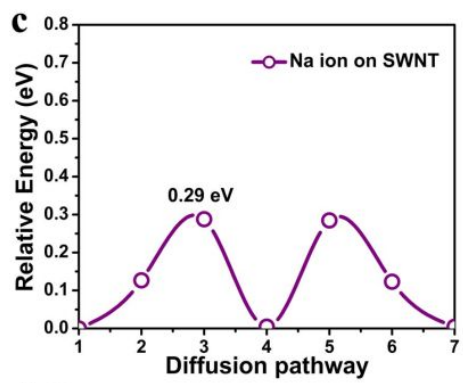

e
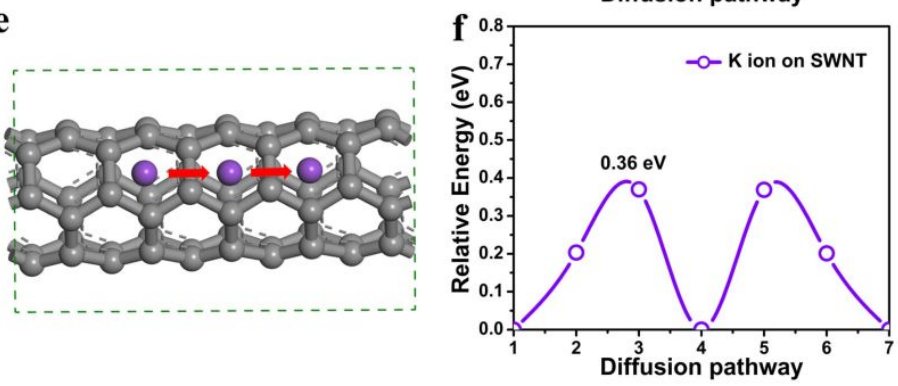

Figure S26. (a, d) Side, (b, e) top views of ion migration path, and (c, f) ion diffusion energy barriers of (a-c) Na ion and (d-f) K ion on SWNT surface. 
$\mathbf{a}$

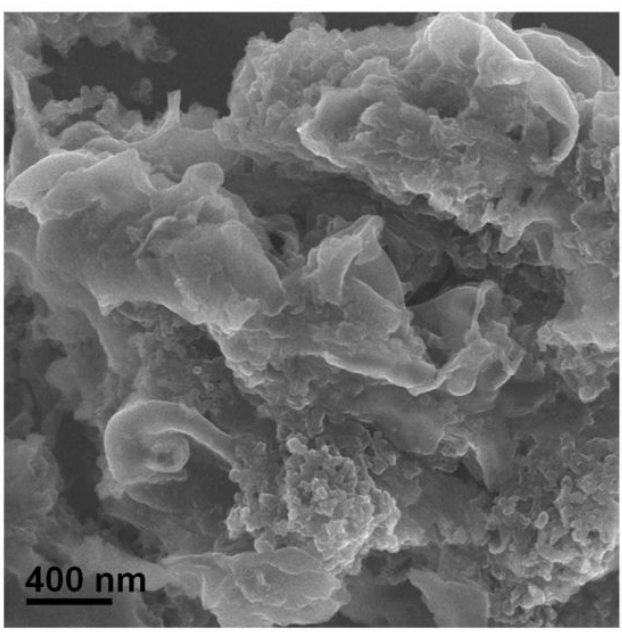

C

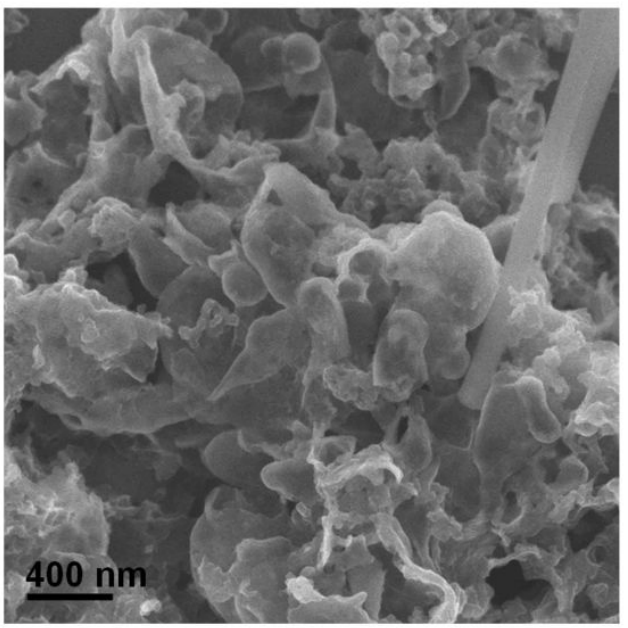

b

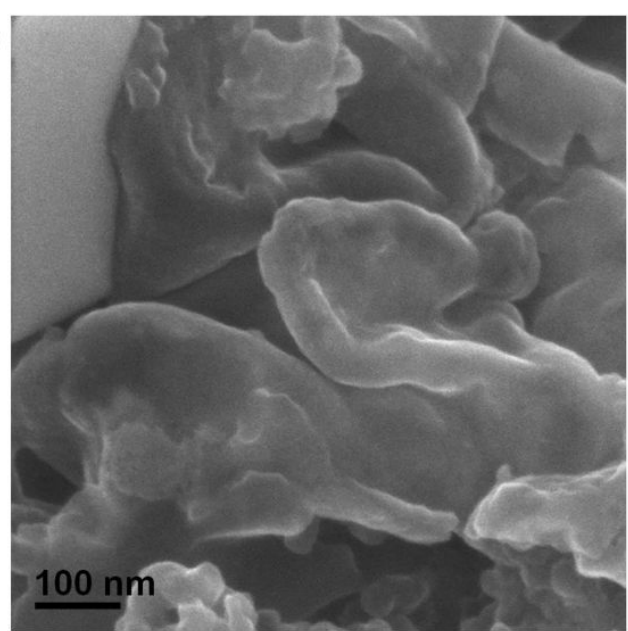

d

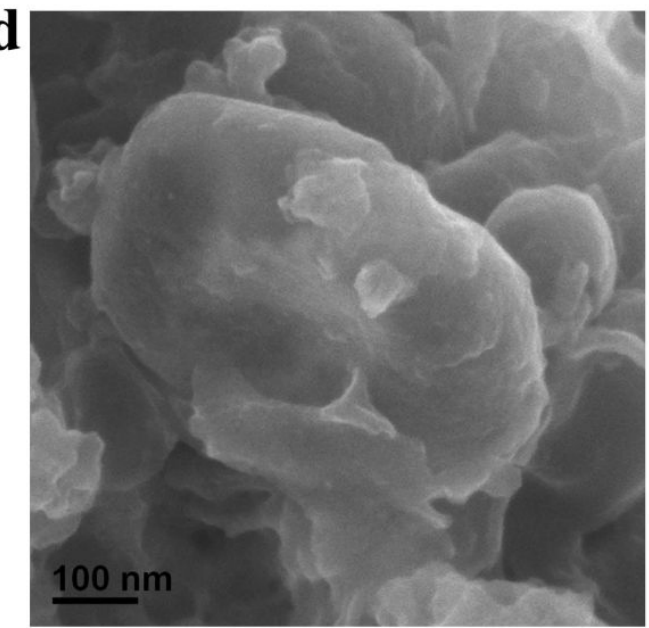

Figure S27. SEM images of $\mathrm{Fe}_{1-\mathrm{x}} \mathrm{S} / \mathrm{SWNT} @ \mathrm{C}$ electrode after 50 cycles for (a-b) SIBs and (c-d) PIBs. It can be seen that the two-dimensional structure was well preserved without the rupture of carbon layer, illustrating its structural integrity during repeated sodiation/desodiation and potassiation/depotassiation cycles. 


\section{References:}

S1. Liu, Y.; Fang, Y. J.; Zhao, Z. W.; Yuan, C. Z.; Lou, X. W., A Ternary $\mathrm{Fe}_{1-\mathrm{x}} \mathrm{S} @$ Porous Carbon Nanowires/Reduced Graphene Oxide Hybrid Film Electrode with Superior Volumetric and Gravimetric Capacities for Flexible Sodium Ion Batteries. Adv. Energy Mater. 2019, 9, 1803052.

S2. Ko, Y. N.; Choi, S. H.; Park, S. B.; Kang, Y. C., Hierarchical MoSe 2 Yolk-Shell Microspheres with Superior Na-Ion Storage Properties. Nanoscale 2014, 6, 10511-10515.

S3. Choi, S. H.; Kang, Y. C., Synergetic Effect of Yolk-Shell Structure and Uniform Mixing of $\mathrm{SnS}-\mathrm{MoS}_{2}$ Nanocrystals for Improved Na-Ion Storage Capabilities. ACS Appl. Mater. Inter. 2015, 7, 24694-24702.

S4. Geng, H.; Yang, J.; Dai, Z.; Zhang, Y.; Zheng, Y.; Yu, H.; Wang, H.; Luo, Z.; Guo, Y.; Zhang, Y.; Fan, H.; Wu, X.; Zheng, J.; Yang, Y.; Yan, Q.; Gu, H., $\mathrm{Co}_{9} \mathrm{~S}_{8} / \mathrm{MoS}_{2}$ Yolk-Shell Spheres for Advanced Li/Na Storage. Small 2017, 13, 1603490.

S5. Wang, Y. X.; Yang, J. P.; Chou, S. L.; Liu, H. K.; Zhang, W. X.; Zhao, D. Y.; Dou, S. X., Uniform Yolk-Shell Iron Sulfide-Carbon Nanospheres for Superior Sodium-Iron Sulfide Batteries. Nat. Commun. 2015, 6, 8689.

S6. Li, H. Z.; Yang, L. Y.; Liu, J.; Li, S. T.; Fang, L. B.; Lu, Y. K.; Yang, H. R.; Liu, S. L.; Lei, M., Improved Electrochemical Performance of Yolk-Shell Structured $\mathrm{SnO}_{2} @$ void@C Porous Nanowires as Anode for Lithium and Sodium Batteries. $J$. Power Sources 2016, 324, 780-787.

S7. Liu, J.; Kopold, P.; Wu, C.; van Aken, P. A.; Maier, J.; Yu, Y., Uniform Yolk-Shell $\mathrm{Sn}_{4} \mathrm{P}_{3} @ \mathrm{C}$ Nanospheres as High-Capacity and Cycle-Stable Anode Materials for Sodium-Ion Batteries. Energ. Environ. Sci. 2015, 8, 3531-3538.

S8. Li, S. T.; Wang, Z. M.; Liu, J.; Yang, L. Y.; Guo, Y.; Cheng, L. Z.; Lei, M.; Wang, W. J., Yolk-Shell Sn@C Eggette-like Nanostructure: Application in Lithium-Ion and Sodium-Ion Batteries. ACS Appl Mater Inter 2016, 8, 19438-19445.

S9. Wang, N. N.; Bai, Z. C.; Qian, Y. T.; Yang, J., One-Dimensional Yolk-Shell $\mathrm{Sb} @$ Ti-O-P Nanostructures as a High-Capacity and High-Rate Anode Material for 
Sodium Ion Batteries. ACS Appl. Mater. Inter. 2017, 9, 447-454.

S10. Ma, L. B.; Yan, P. J.; Wu, S. K.; Zhu, G. Y.; Shen, Y. L., Engineering Tin

Phosphides@Carbon Yolk-Shell Nanocube Structures as a Highly Stable Anode Material for Sodium-Ion Batteries. J. Mater. Chem. A 2017, 5, 16994-17000.

S11. Liu, Z. M.; Lu, T. C.; Song, T.; Yu, X. Y.; Lou, X. W.; Paik, U.,

Structure-Designed Synthesis of $\mathrm{FeS}_{2} @ \mathrm{C}$ Yolk-Shell Nanoboxes as a High-Performance Anode for Sodium-Ion Batteries. Energy Environ. Sci, 2017, 10, $1576-1580$.

S12. Liu, J.; Yu, L. T.; Wu, C.; Wen, Y. R.; Yin, K. B.; Chiang, F. K.; Hu, R. Z.;

Liu, J. W.; Sun, L. T.; Gu, L.; Maier, J.; Yu, Y.; Zhu, M., New Nanoconfined Galvanic Replacement Synthesis of Hollow Sb@C Yolk-Shell Spheres Constituting a Stable Anode for High-Rate Li/Na-Ion Batteries. Nano Lett. 2017, 17, 2034-2042.

S13. $\quad$ Song, J. H.; Yan, P. F.; Luo, L. L.; Qi, X. G.; Rong, X. H.; Zheng, J. M.; Xiao, B. W.; Feng, S.; Wang, C. M.; Hu, Y. S.; Lin, Y. H.; Sprenkle, V. L.; Li, X. L., Yolk-Shell Structured Sb@C Anodes for High Energy Na-Ion Batteries. Nano Energy 2017, 40, 504-511.

S14. Wu, Y. Z.; Meng, J. S.; Li, Q.; Niu, C. J.; Wang, X. P.; Yang, W.; Li, W.; Mai, L. Q., Interface-Modulated Fabrication of Hierarchical Yolk-Shell $\mathrm{Co}_{3} \mathrm{O}_{4} / \mathrm{C}$ Dodecahedrons as Stable Anodes for Lithium and Sodium Storage. Nano Res. 2017, 10, 2364-2376.

S15. Li, Q. D.; Li, L.; Owusu, K. A.; Luo, W.; An, Q. Y.; Wei, Q. L.; Zhang, Q. J.; Mai, L. Q., Self-Adaptive Mesoporous CoS@Alveolus-Like Carbon Yolk-Shell Microsphere for Alkali Cations Storage. Nano Energy 2017, 41, 109-116.

S16. Veerasubramani, G. K.; Subramanian, Y.; Park, M. S.; Nagaraju, G.; Senthilkumar, B.; Lee, Y. S.; Kim, D. W., Enhanced Storage Ability by Using a Porous Pyrrhotite@N-Doped Carbon Yolk-Shell Structure as an Advanced Anode Material for Sodium-Ion Batteries. J. Mater. Chem. A 2018, 6, 20056-20068.

S17. Liu, H.; Liu, B. H.; Guo, H.; Liang, M. F.; Zhang, Y. H.; Borjigin, T.; Yang, X. F.; Wang, L.; Sun, X. L., N-Doped C-Encapsulated Sale-Like Yolk-Shell Frame Assembled by Expanded Planes Few-Layer $\mathrm{MoSe}_{2}$ for Enhanced Performance in 
Sodium-Ion Batteries. Nano Energy 2018, 51, 639-648.

S18. Li, Z.; Fang, Y. J.; Zhang, J. T.; Lou, X. W., Necklace-Like Structures Composed of $\mathrm{Fe}_{3} \mathrm{~N} @ \mathrm{C}$ Yolk-Shell Particles as an Advanced Anode for Sodium-Ion Batteries. Adv. Mater. 2018, 30, 1800525.

S19. Ren, X. C.; Ren, Z. G.; Li, Q. W.; Wen, W.; Li, X. F.; Chen, Y.; Xie, L.; Zhang, L.; Zhu, D. M.; Gao, B.; Chu, P. K.; Huo, K. F., Tailored Plum Pudding-Like $\mathrm{Co}_{2} \mathrm{P} / \mathrm{Sn}$ Encapsulated with Carbon Nanobox Shell as Superior Anode Materials for High-Performance Sodium-Ion Capacitors. Adv. Energy Mater. 2019, 9, 1900091.

S20. Zheng, J. L.; Huang, X. M.; Pan, X.; Teng, C.; Wang, N., Yolk-Shelled $\mathrm{Ni}_{2} \mathrm{P} @$ Carbon Nanocomposite as High-Performance Anode Material for Lithium and Sodium Ion Batteries. Appl. Surf. Sci. 2019, 473, 699-705.

S21. Zhao, Y.; Fu, Q.; Wang, D.; Pang, Q.; Gao, Y.; Missiul, A.; Nemausat, R.; Sarapulova, A.; Ehrenberg, H.; Wei, Y.; Chen, G., Co $9 \mathrm{~S}_{8} @$ Carbon Yolk-Shell Nanocages as a High Performance Direct Conversion Anode Material for Sodium Ion Batteries. Energy Storage Materials 2019, 18, 51-58.

S22. Zang, R.; Li, P.; Guo, X.; Man, Z.; Zhang, S.; Wang, C.; Wang, G., Yolk-Shell N-Doped Carbon Coated $\mathrm{FeS}_{2}$ Nanocages as a High-Performance Anode for Sodium-Ion Batteries. J. Mater. Chem. A 2019, 7, 14051.

S23. Chen, K. Y.; Zhang, W. X.; Xue, L. H.; Chen, W. L.; Xiang, X. H.; Wan, M.; Huang, Y. H., Mechanism of Capacity Fade in Sodium Storage and the Strategies of Improvement for $\mathrm{FeS}_{2}$ Anode. ACS Appl. Mater. Inter. 2017, 9, 1536-1541.

S24. Li, Q. D.; Wei, Q. L.; Zuo, W. B.; Huang, L.; Luo, W.; An, Q. Y.; Pelenovich, V. O.; Mai, L. Q.; Zhang, Q. J., Greigite $\mathrm{Fe}_{3} \mathrm{~S}_{4}$ as a New Anode Material for High-Performance Sodium-Ion Batteries. Chem. Sci. 2017, 8, 160-164.

S25. Wei, X.; Li, W. H.; Shi, J. A.; Gu, L.; Yu, Y., FeS@C on Carbon Cloth as Flexible Electrode for Both Lithium and Sodium Storage. ACS Appl. Mater. Inter. 2015, 7, 27804-27809.

S26. Chen, W. H.; Qi, S. H.; Guan, L. Q.; Liu, C. T.; Cui, S. Z.; Shen, C. Y.; Mi, L. W., Pyrite $\mathrm{FeS}_{2}$ Microspheres Anchoring on Reduced Graphene Oxide Aerogel as an Enhanced Electrode Material for Sodium-Ion Batteries. J. Mater. Chem. A 2017, 5, 
$5332-5341$

S27. Chen, Y. Y.; Hu, X. D.; Evanko, B.; Sun, X. H.; Li, X.; Hou, T. Y.; Cai, S.;

Zheng, C. M.; Hu, W. B.; Stucky, G. D., High-Rate FeS $_{2} / C N T$ Neural Network Nanostructure Composite Anodes for Stable, High-Capacity Sodium-Ion Batteries. Nano Energy 2018, 46, 117-127.

S28. Lee, S. Y.; Kang, Y. C., Sodium-Ion Storage Properties of FeS-Reduced Graphene Oxide Composite Powder with a Crumpled Structure. Chem. Eur. J. 2016, $22,2769-2774$.

S29. Cao, Z. J.; Song, H. H.; Cao, B.; Ma, J.; Chen, X. H.; Zhou, J. S.; Ma, Z. K., Sheet-on-Sheet Chrysanthemum-Like C/FeS Microspheres Synthesized by One-Step Solvothermal Method for High-Performance Sodium-Ion Batteries. J. Power Sources 2017, 364, 208-214.

S30. Tan, Y. Z.; Wong, K. W.; Zhang, Z. L.; Ng, K. M., In Situ Synthesis of Iron Sulfide Embedded Porous Carbon Hollow Spheres for Sodium Ion Batteries. Nanoscale 2017, 9, 19408-19414.

S31. Li, L. L.; Peng, S. J.; Bucher, N.; Chen, H. Y.; Shen, N.; Nagasubramanian, A.; Eldho, E.; Hartung, S.; Ramakrishna, S.; Srinivasan, M., Large-Scale Synthesis of Highly Uniform $\mathrm{Fe}_{1-\mathrm{x}} \mathrm{S}$ Nanostructures as a High-Rate Anode for Sodium Ion Batteries. Nano Energy 2017, 37, 81-89.

S32. Xiao, Y.; Hwang, J. Y.; Belharouak, I.; Sun, Y. K., Na Storage Capability Investigation of a Carbon Nanotube-Encapsulated $\mathrm{Fe}_{1-\mathrm{x}} \mathrm{S}$ Composite. ACS Energy Lett. 2017, 2, 364-372.

S33. Choi, M. J.; Kim, J.; Yoo, J. K.; Yim, S.; Jeon, J.; Jung, Y. S., Extremely Small Pyrrhotite $\mathrm{Fe}_{7} \mathrm{~S}_{8}$ Nanocrystals with Simultaneous Carbon-Encapsulation for High-Performance Na-Ion Batteries. Small 2018, 14, 1702816.

S34. Wang, Q. H.; Guo, C.; Zhu, Y. X.; He, J. P.; Wang, H. Q., Reduced Graphene Oxide-Wrapped $\mathrm{FeS}_{2}$ Composite as Anode for High-Performance Sodium-Ion Batteries. Nano-Micro Lett. 2018, 10, 30.

S35. Huang, W.; Sun, H. Y.; Shangguan, H. H.; Cao, X. Y.; Xiao, X. X.; Shen, F.; Molhave, K.; Ci, L. J.; Si, P. C.; Zhang, J. D., Three-Dimensional Iron 
Sulfide-Carbon Interlocked Graphene Composites for High-Performance Sodium-Ion Storage. Nanoscale 2018, 10, 7851-7859.

S36. Bu, F. X.; Xiao, P. T.; Chen, J. D.; Aboud, M. F. A.; Shakir, I.; Xu, Y. X., Rational Design of Three-Dimensional Graphene Encapsulated Core-Shell FeS@Carbon Nanocomposite as a Flexible High-Performance Anode for Sodium-Ion Batteries. J. Mater. Chem. A 2018, 6, 6414-6421.

S37. Zhu, X. X.; Liu, D.; Zheng, D.; Wang, G. W.; Huang, X. K.; Harris, J. S.; Qu, D. Y.; Qu, D. Y., Dual Carbon-Protected Metal Sulfides and Their Application to Sodium-Ion Battery Anodes. J. Mater. Chem. A 2018, 6, 13294-13301.

S38. Xie, J. P.; Zhu, Y. Q.; Zhuang, N.; Lei, H.; Zhu, W. L.; Fu, Y.; Javed, M. S.; Li, J. L.; Mai, W. J., Rational Design of Metal Organic Framework-Derived $\mathrm{FeS}_{2}$ Hollow Nanocages@Reduced Graphene Oxide for K-Ion Storage. Nanoscale 2018, 10, 17092-17098.

S39. Zhao, Y.; Zhu, J. J.; Ong, S. J. H.; Yao, Q. Q.; Shi, X. L.; Hou, K.; Xu, Z. C. J.; Guan, L. H., High-Rate and Ultralong Cycle-Life Potassium Ion Batteries Enabled by In Situ Engineering of Yolk-Shell FeS $@$ @ Structure on Graphene Matrix. Adv Energy Mater 2018, 8, 1802565.

S40. Zhao, Z.; Hu, Z.; Jiao, R.; Tang, Z.; Dong, P.; Li, Y.; Li, S.; Li, H., Tailoring Multi-Layer Architectured $\mathrm{FeS}_{2} @ \mathrm{C}$ Hybrids for Superior Sodium-, Potassium- and Aluminum-Ion Storage. Energy Storage Materials 2019, 22, 228-234.

S41. Chen, C. M.; Yang, Y. C.; Tang, X.; Qiu, R. H.; Wang, S. Y.; Cao, G. Z.; Zhang, M., Graphene-Encapsulated $\mathrm{FeS}_{2}$ in Carbon Fibers as High Reversible Anodes for $\mathrm{Na}^{+} / \mathrm{K}^{+}$Batteries in a Wide Temperature Range. Small 2019, 15, 1804740.

S42. Jian, Z. L.; Luo, W.; Ji, X. L., Carbon Electrodes for K-Ion Batteries. J. Am. Chem. Soc. 2015, 137, 11566-11569.

S43. Xie, Y. H.; Chen, Y.; Liu, L.; Tao, P.; Fan, M. P.; Xu, N.; Shen, X. W.; Yan, C. L., Ultra-High Pyridinic N-Doped Porous Carbon Monolith Enabling High-Capacity K-Ion Battery Anodes for Both Half-Cell and Full-Cell Applications. Adv. Mater. 2017, 29, 1702268.

S44. Yang, J. L.; Ju, Z. C.; Jiang, Y.; Xing, Z.; Xi, B. J.; Feng, J. K.; Xiong, S. L., 
Enhanced Capacity and Rate Capability of Nitrogen/Oxygen Dual-Doped Hard Carbon in Capacitive Potassium-Ion Storage. Adv. Mater. 2018, 30, 1700104.

S45. Mahmood, A.; Li, S.; Ali, Z. S.; Tabassum, H.; Zhu, B. J.; Liang, Z. B.; Meng, W.; Aftab, W.; Guo, W. H.; Zhang, H.; Yousaf, M.; Gao, S.; Zou, R. Q.; Zhao, Y. S., Ultrafast Sodium/Potassium-Ion Intercalation into Hierarchically Porous Thin Carbon Shells. Adv. Mater. 2019, 31, 1805430.

S46. Lakshmi, V.; Chen, Y.; Mikhaylov, A. A.; Medvedev, A. G.; Sultana, I.; Rahman, M. M.; Lev, O.; Prikhodchenko, P. V.; Glushenkov, A. M., Nanocrystalline $\mathrm{SnS}_{2}$ Coated onto Reduced Graphene Oxide: Demonstrating the Feasibility of a Non-Graphitic Anode with Sulfide Chemistry for Potassium-Ion Batteries. Chem. Commun. 2017, 53, 8272-8275.

S47. Zhou, J. H.; Wang, L.; Yang, M. Y.; Wu, J. H.; Chen, F. J.; Huang, W. J.; Han, N.; Ye, H. L.; Zhao, F. P.; Li, Y. Y.; Li, Y. G., Hierarchical VS 2 Nanosheet Assemblies: A Universal Host Material for the Reversible Storage of Alkali Metal Ions. Adv. Mater. 2017, 29, 1702061.

S48. Sultana, I.; Rahman, M. M.; Mateti, S.; Ahmadabadi, V. G.; Glushenkov, A. M.; Chen, Y., K-Ion and Na-Ion Storage Performances of $\mathrm{Co}_{3} \mathrm{O}_{4}-\mathrm{Fe}_{2} \mathrm{O}_{3}$ Nanoparticle-Decorated Super P Carbon Black Prepared by a Ball Milling Process. Nanoscale 2017, 9, 3646-3654.

S49. Lu, Y. Y.; Chen, J., Robust Self-Supported Anode by Integrating $\mathrm{Sb}_{2} \mathrm{~S}_{3}$ Nanoparticles with S,N-Codoped Graphene to Enhance K-Storage Performance. Sci. China Chem. 2017, 60, 1533-1539.

S50. Xie, K. Y.; Yuan, K.; Li, X.; Lu, W.; Shen, C.; Liang, C. L.; Vajtai, R.; Ajayan, P.; Wei, B. Q., Superior Potassium Ion Storage via Vertical $\mathrm{MoS}_{2}$ "Nano-Rose" with Expanded Interlayers on Graphene. Small 2017, 13, 1701471.

S51. Gao, H.; Zhou, T. F.; Zheng, Y.; Zhang, Q.; Liu, Y. Q.; Chen, J.; Liu, H. K.; Guo, Z. P., CoS Quantum Dot Nanoclusters for High-Energy Potassium-Ion Batteries. Adv. Funct. Mater. 2017, 27, 1702634.

S52. Zhang, W. C.; Mao, J. F.; Li, S. A.; Chen, Z. X.; Guo, Z. P., Phosphorus-Based Alloy Materials for Advanced Potassium-Ion Battery Anode. J. 
Am. Chem. Soc. 2017, 139, 3316-3319.

S53. Mao, M. L.; Cui, C. Y.; Wu, M. G.; Zhang, M.; Gao, T.; Fan, X. L.; Chen, J.;

Wang, T. H.; Ma, J. M.; Wang, C. S., Flexible $\mathrm{ReS}_{2}$ Nanosheets/N-Doped Carbon Nanofibers-Based Paper as a Universal Anode for Alkali (Li, Na, K) Ion Battery. Nano Energy 2018, 45, 346-352.

S54. Liu, Z. W.; Li, P.; Suo, G. Q.; Gong, S.; Wang, W.; Lao, C. Y.; Xie, Y. J.; Guo, H.; Yu, Q. Y.; Zhao, W.; Han, K.; Wang, Q.; Qin, M. L.; Xi, K.; Qu, X. H., Zero-Strain $\mathrm{K}_{0.6} \mathrm{Mn}_{1} \mathrm{~F}_{2.7}$ Hollow Nanocubes for Ultrastable Potassium Ion Storage. Energy Environ. Sci. 2018, 11, 3033-3042.

S55. Yu, Q. Y.; Jiang, B.; Hu, J.; Lao, C. Y.; Gao, Y. Z.; Li, P. H.; Liu, Z. W.; Suo, G. Q.; He, D. L.; Wang, W.; Yin, G. P., Metallic Octahedral CoSe 2 Threaded by N-Doped Carbon Nanotubes: A Flexible Framework for High-Performance Potassium-Ion Batteries. Adv. Sci. 2018, 5, 1800782.

S56. Wang, W.; Jiang, B.; Qian, C.; Lv, F.; Feng, J. R.; Zhou, J. H.; Wang, K.; Yang, C.; Yang, Y.; Guo, S. J., Pistachio-Shuck-Like $\mathrm{MoSe}_{2} / \mathrm{C}$ Core/Shell Nanostructures for High-Performance Potassium-Ion Storage. Adv. Mater. 2018, 30, 1801812.

S57. Bai, J.; Xi, B. J.; Mao, H. Z.; Lin, Y.; Ma, X. J.; Feng, J. K.; Xiong, S. L., One-Step Construction of N,P-Codoped Porous Carbon Sheets/CoP Hybrids with Enhanced Lithium and Potassium Storage. Adv. Mater. 2018, 30, 1802310.

S58. Jia, B. R.; Yu, Q. Y.; Zhao, Y. Z.; Qin, M. L.; Wang, W.; Liu, Z. W.; Lao, C. Y.; Liu, Y.; Wu, H. W.; Zhang, Z. L.; Qu, X. H., Bamboo-Like Hollow Tubes with $\mathrm{MoS}_{2} / \mathrm{N}-$ Doped-C Interfaces Boost Potassium-Ion Storage. Adv. Funct. Mater. 2018, $28,1803409$.

S59. Ge, J. M.; Fan, L.; Wang, J.; Zhang, Q. F.; Liu, Z. M.; Zhang, E. J.; Liu, Q.; Yu, X. Z.; Lu, B. A., MoSe 2 /N-Doped Carbon as Anodes for Potassium-Ion Batteries. Adv. Energy Mater. 2018, 8, 1801477.

S60. Huang, H. W.; Cui, J.; Liu, G. X.; Bi, R.; Zhang, L., Carbon-Coated $\mathrm{MoSe}_{2} / \mathrm{MXene}$ Hybrid Nanosheets for Superior Potassium Storage. ACS Nano 2019, $13,3448-3456$. 
S61. Yang, F. H.; Goo, H.; Hao, J. N.; Zhang, S. L.; Li, P.; Liu, Y. Q.; Chen, J.; Guo, Z. P., Yolk-Shell Structured FeP@C Nanoboxes as Advanced Anode Materials for Rechargeable Lithium-/Potassium-Ion Batteries. Adv. Funct. Mater. 2019, 29, 1808291.

S62. Li, D.; Sun, Q.; Zhang, Y.; Chen, L.; Wang, Z.; Liang, Z.; Si, P.; Ci, L., Surface-Confined SnS $\mathrm{S}_{2} @ \mathrm{C} @ \mathrm{rGO}$ as High-Performance Anode Materials for Sodiumand Potassium-Ion Batteries. Chemsuschem 2019, 12, 2689-2700.

S63. Wang, J.; Fan, L.; Liu, Z. M.; Chen, S. H.; Zhang, Q. F.; Wang, L. L.; Yang, H. G.; Yu, X. Z.; Lu, B. A., In Situ Alloying Strategy for Exceptional Potassium Ion Batteries. ACS Nano 2019, 13, 3703-3713.

S64. Tan, Q. W.; Li, P.; Han, K.; Liu, Z. W.; Li, Y.; Zhao, W.; He, D. L.; An, F. Q.; Qin, M. L.; Qu, X. H., Chemically Bubbled Hollow Fe $\mathrm{X}_{\mathrm{X}}$ Nanospheres Anchored on 3D N-Doped Few-Layer Graphene Architecture as a Performance-Enhanced Anode Material for Potassium-Ion Batteries. J. Mater. Chem. A 2019, 7, 744-754.

S65. Ge, P.; Hou, H. S.; Li, S. J.; Yang, L.; Ji, X. B., Tailoring Rod-Like FeSe 2 Coated with Nitrogen-Doped Carbon for High-Performance Sodium Storage. Adv. Funct. Mater. 2018, 28, 1801765.

S66. Li, J. X.; Zou, M. Z.; Zhao, Y.; Lin, Y. B.; Lai, H.; Guan, L. H.; Huang, Z. G., Coaxial MWNTs@ $\mathrm{MnO}_{2}$ Confined in Conducting PPy for Kinetically Efficient and Long-Term Lithium Ion Storage. Electrochim Acta 2013, 111, 165-171.

S67. Hou, B. H.; Wang, Y. Y.; Guo, J. Z.; Ning, Q. L.; Xi, X. T.; Pang, W. L.; Cao, A. M.; Wang, X. L.; Zhang, J. P.; Wu, X. L., Pseudocapacitance-Boosted Ultrafast Na Storage in a Pie-Like FeS@C Nanohybrid as an Advanced Anode Material for Sodium-Ion Full Batteries. Nanoscale 2018, 10, 9218-9225.

S68. Ding, N.; Xu, J.; Yao, Y. X.; Wegner, G.; Fang, X.; Chen, C. H.; Lieberwirth, I., Determination of the Diffusion Coefficient of Lithium Ions in Nano-Si. Solid State Ionics 2009, 180, 222-225.

S69. Yan, Y.; Ben, L. B.; Zhan, Y. J.; Huang, X. J., Nano-Sn Embedded in Expanded Graphite as Anode for Lithium Ion Batteries with Improved Low Temperature Electrochemical Performance. Electrochim Acta 2016, 187, 186-192. 
S70. Zhao, Y.; Wang, L. P.; Xi, S. B.; Du, Y. H.; Yao, Q. Q.; Guan, L. H.; Xu, Z. C. J., Encapsulating Porous $\mathrm{SnO}_{2}$ into a Hybrid Nanocarbon Matrix for Long Lifetime Li Storage. J. Mater. Chem. A 2017, 5, 25609-25617.

S71. Xu, Y.; Zhang, C. L.; Zhou, M.; Fu, Q.; Zhao, C. X.; Wu, M. H.; Lei, Y., Highly Nitrogen Doped Carbon Nanofibers with Superior Rate Capability and Cyclability for Potassium Ion Batteries. Nat. Commun. 2018, 9, 1720. 